\title{
Exploring the Stochastic Host-Pathogen Tuberculosis Model with Adaptive Immune Response
}

\author{
S. P. Rajasekar, ${ }^{1,2}$ M. Pitchaimani, ${ }^{1}$ Quanxin Zhu $\mathbb{D O}^{3}$ and Kaibo Shi ${ }^{4}$ \\ ${ }^{1}$ Ramanujan Institute for Advanced Study, in Mathematics, University of Madras, Chennai 600 005, Tamil Nadu, India \\ ${ }^{2}$ Department of Mathematics, Government Arts College for Women, Nilakottai 624 202, Tamil Nadu, India \\ ${ }^{3}$ MOE-LCSM, School of Mathematics and Statistics, Hunan Normal University, Changsha 410081, China \\ ${ }^{4}$ School of Information Science and Engineering, Chengdu University, Chengdu 610106, China \\ Correspondence should be addressed to Quanxin Zhu; zqx22@126.com
}

Received 18 September 2020; Revised 14 December 2020; Accepted 9 May 2021; Published 19 June 2021

Academic Editor: Juan P. Amezquita-Sanchez

Copyright $\odot 2021$ S. P. Rajasekar et al. This is an open access article distributed under the Creative Commons Attribution License, which permits unrestricted use, distribution, and reproduction in any medium, provided the original work is properly cited.

\begin{abstract}
In this literature, we probe a stochastic host-pathogen tuberculosis model with the adaptive immune response of four states of epidemiological classification: Mycobacterium tuberculosis, uninfected macrophages, infected macrophages, and immune response CD4+ T cells. This model is pertinent to the latent stage of tuberculosis infection and active tuberculosis-infected individuals. The stochastic host-pathogen tuberculosis model in pathology is constituted based on the environmental influence on the Mycobacterium tuberculosis and macrophage population, elucidated by stochastic perturbations, and it is proportional to each state. We evince the existence and a unique global positive solution of a stochastic tuberculosis model. We attain sufficient conditions for the extinction of the tubercle bacillus. Moreover, we acquire the existence of the stationary distribution of the positive solutions by the Lyapunov function method. Eventually, numerical simulations validate analytical findings and the dynamics of the stochastic TB model.
\end{abstract}

\section{Introduction}

In the annals of history, there have been many threats such as natural calamity, diseases, and wild animals for the existence of humans; among these, diseases seem to be the main cause of death. In the previous centuries, tuberculosis posed a great threat to the survival of human beings. It is believed that it was the cause of death of more than a third population [1]. And block death in Europe is the standing testimony for these facts. Fortunately, due to the development of medical science, tuberculosis is completely curable but still persists.

Tuberculosis is traced back to $3400-2400$ BC. It is as ancient as that of ancient countries such as Egypt, Greece, Rome, and India. The German Nobel laureate Robert Koch (1843-1910) discovered the tubercle bacillus (March 24, 1882 ) at the backdrop of one in seven who died in Europe due to mysterious disease.

Infection of the lungs bacteria (Mycobacterium tuberculosis (MTB)) leads to tuberculosis (TB). The bacterial infection may also spread into the brain, kidneys, spine, etc., but it is fortunate that tuberculosis is curable and preventable [1]. Tuberculosis is contagious and spreads through the air. Even the inhalation of a few of these germs is potential enough to infect a person. The physical contact, the nearness, and the air around the infected person can also infect. Sharing the same space with the infected person, weak immunity, malnutrition, unemployment, poor lifestyle, poor food habits, lack of shelter, unhygienic environment, drug abuse, imperfect sexual attitude, ill-literacy, and smoking are vulnerable factors for the spread of tuberculosis [2].

Infection is mainly occurred by the aerosol route like inhalation, wherein bacilli contained droplet nuclei sets in the alveoli. Here, alveolar macrophages [3] engulf the bacteria and ultimately bring out the infection to dendritic cells and all other cells as epithelial cells. These alveolar macrophages possess multiple microbicidal mechanisms which include phagolysosome fusion and respiratory burst to prevent postinfective mechanisms. For successful 
sustainability of infection, the tuberculosis bacilli must understand its encounter with the collection of activated macrophages (granuloma) $[4,5]$ and eventually gain access to the lymphocytes, neutrophils, fibroblasts, eosinophils, or the bloodstream $[6,7]$. Macrophages with the potential to sterile and kill the bacteria are segmented apart in the tissue. In the case of adaptive immunity with the accumulation of effector CD4+ and CD8+ $\mathrm{T}$ cells setting in lungs, the multiplication of the bacterial population is contained and enumerable patients become asymptomatic and unable to shed bacteria, and they are then considered as vulnerable to latent TB infection (LTBI). Varied mechanisms cause the limited ability with adaptive immune responses to encounter and eradicate MTB, some of which are then categorized and characterized such as weakened histocompatibility complex (MHC) class II-mediated antigen presentation; adaptation of the anti-inflammatory mediator lipoxin A4; containing by regulatory $\mathrm{T}$ cells; monitoring bacterial antigen gene expression and hence incapable of reducing antigen-specific CD4+ T cells; and immune to the macrophages activating effector cytokines such as interferon- $\gamma($ INF- $\gamma$ ) and tumour necrosis factor- $\alpha$ (TNF- $\alpha)[4,8]$.

The latent stage of the tuberculosis infection is nonchallenged; though the germs are present in the body, they are inactive and harmless. Antibiotics such as isoniazid (INH), rifampin (Rifadin, Rimactane), and rifapentine can also be used to contain the aggravation of the germs at this stage. It is very easy to wipe out the germs from the body at this stage. Indications of active tuberculosis infection are coughing, sudden weight loss, loss of appetite, sweating during nights, fever, and chest pain.

The potential roles for adjunctive immunotherapy in multidrug-resistant TB (MDR-TB) and extensively drugresistant TB (XDR-TB) [9-11] consist in enhancing cure rates and reducing time spent to cultural shifts, reducing the tissue damage concerned with nonproductive postimmune reactions, and elevating overall health status by subdoing systematic impacts of prolonged chronic inflammation. In addition, enumerable powerfully useful cytokines and other immunomodulatory agents already exist in medical use for other conditions.

A significant role in the immunity against pathogen agent is played by $\mathrm{T}$-cell differentiation and memory and effector T cells [12-14]. Hence, an effective T-cell response decides if the infection decreases or develops into a medically evident disease. Studies prove that CD4+ T cells [13-15] do their part in the protection against MTB as strengthened by the evidence that CD4+ T cells such as T helper 1 (Th1), Th2, Th17, and regulatory T cells (Tregs) and all these subsets support one another to restrict infection. MTB-specific CD4+ Th1 cell response is recognized as possessing a protective role for the power to generate cytokines such as INF- $\gamma$ and TNF- $\alpha$ that involve the processes of recruitment and activation of innate immune cells as monocytes and granulocytes. In this way, when other antigen-specific T cells such as CD8+ cells [14], natural killer (NK) cells, $\gamma \delta \mathrm{T}$ cells, nonclassical (MHC) class I molecule CD1, controlled T cells are able to raise INF- $\gamma$ during MTB infection and they are unable to equalize the lack of CD4+ T cells.

Of late, studies have revealed that multifunctional/polyfunctional $\mathrm{T}$ cells (i.e., $\mathrm{T}$ cells possessed with multiple effector functions) $[13,14]$ can produce IFN- $\gamma$ coupled with IL-2, and subsequently, a subset of cells is able to concurrently produce IFN- $\gamma$, TNF- $\alpha$, IL-2, and IL-17 and was diagnosed in patients with active TB disease compared to LTBI, which rapidly decreased on anti-TB treatment. And also, the polyfunctional $\mathrm{T}$ cells were recognized for their potentiality to proliferate and to secrete very many cytokines, and these cells were discovered to act a protective role in antiviral immunity in chronic infections (when the antigen load is low).

Memory $\mathrm{T}$ cells are a subset of infection-fighting $\mathrm{T}$ cells (T lymphocytes) that are highly proliferative and possess a strong immune response. Based on the role of L-selectin and CC-chemokine receptor 7 , memory T cells can be segmented into $\mathrm{CD}^{2} \mathrm{~L}^{\text {hi }} \mathrm{CCR7}^{+}$and $\mathrm{CD}^{2} \mathrm{~L}^{\text {lo }} \mathrm{CCR}^{-}$[12-14]. In the case of in vitro stimulation, humanCD62 $\mathrm{L}^{\text {hi }} \mathrm{CCR} 7^{+}$memory CD4+T cells caused the production of IL-2 but hardly produce interferon- $\gamma$, IL-4, or IL-5. But CD62L ${ }^{\text {lo }}$ CCR7 $^{-}$ cells rapidly produced these effector cytokines but produced little IL-2. CCR7 ${ }^{+}$cells named as central memory $\left(T_{\mathrm{CM}}\right)$ cells $[13,14]$ are potential enough to produce numerous IL-2 but low lying of effector cytokines and home to secondary lymphoid tissues. But CCR7 ${ }^{-}$cells termed as effector memory $\left(T_{\mathrm{EM}}\right)$ cells [14] are capable of producing high order of cytokines and home to peripheral tissues. Subsequently, there are various other subpopulations of memory $\mathrm{T}$ cells such as memory $\mathrm{T}$ stem cells $\left(T_{\mathrm{SCM}}\right.$, multiple stem-like properties), $\mathrm{T}$ naive phenotype $\left(T_{N}\right)$, and transitional memory $\mathrm{T}$ cells $\left(T_{\mathrm{TM}}\right)[13,14]$, very many of which were secreted in the peripheral blood of healthy individuals.

Regulatory T cells $\left(T_{\text {reg }}\right)$ [16] are inevitable for monitoring peripheral tolerance, preventing self-generating immunity, and reducing chronic inflammatory disease. In fact, $T_{\text {reg }}$ have varied mechanisms within their powers, and one such mechanism is the suppression by inhibitory cytokines which include IL-10, transforming (TGF- $\beta$ ), and latest identified IL-35 which are prime mediators of $T_{\text {reg- }}$ cell function. CD4+CD25+FOXP3 + circulating $T_{\text {reg }}$ play a primary role in restricting immune responses by downregulating $\mathrm{CD} 4+$ or CD8+ cell functions. Hectic suppression by $T_{\text {reg }}$ is important in regulating immune responses against MTB.

In spite of the fact that the role of CD8 T cells [13] in TB is less clear than that of CD4 T cells, they are considered in general to impart optimal immunity and protection. CD8 $\mathrm{T}$ cells contain enumerable antimicrobial effector mechanisms that are less vital or cease to exist in CD4 Th1 and Th17 T cells. CD8 T cells emit cytokines or cytotoxic molecules, which cause apoptosis of target cells. Th1 CD4 T cells propel effector functions in macrophages that contain intracellular MTB, and their role has been monitored with protection. Besides, many studies have depicted that Th17 cells, which can produce IL-17, are implicated in immune protection against MTB, mainly due to the effect of this 
cytokine in incurring and vibrating neutrophils. Th 17 cells have been implicated in the protection against TB at initial levels, for their power to employ monocytes and Th1 lymphocytes to the space of granuloma formation [5]. Contrarily, very many studies have manifested that unrestricted Th17 stimulation confirms an exaggerated inflammation catalyzed by neutrophils and inflammatory monocytes that rush to the place of disease during the damage of tissue.

MTB-specific CD4+ T-cell protective response is simply due to Th1 cells and is negotiated by IFN- $\gamma$ and TNF- $\alpha$ that employ monocytes and granulocytes and enhance their antimicrobial attitudes. Subsequently, higher part of antigenspecific effector memory cells and diminished frequency of central memory CD4+ Tcells have been discovered in patients with active TB in comparison with distribution witness in LTBI individuals. All the information converges on the increase of this complicated disease with the aim of improving diagnosis, prognosis, drug therapy, and vaccination.

Zhang [17] acclaimed the following host tuberculosis model:

$$
\begin{aligned}
\frac{\mathrm{d} B}{\mathrm{~d} t} & =\delta B\left(1-\frac{B}{K}\right)+M_{i}\left(\eta_{1} b+\eta_{2} \gamma \frac{T}{c+T}\right)-M_{u} B\left(\zeta+\eta_{3} \beta\right), \\
\frac{\mathrm{d} M_{u}}{\mathrm{~d} t} & =s_{M}-\mu_{M} M_{u}-\beta M_{u} B, \\
\frac{\mathrm{d} M_{i}}{\mathrm{~d} t} & =\beta M_{u} B-b M_{i}-\gamma M_{i} \frac{T}{c+T} \\
\frac{\mathrm{d} T}{\mathrm{~d} t} & =s_{T}+c_{M} M_{i} \frac{T}{1+e_{m} T}+c_{B} B \frac{T}{1+e_{B} T}-\mu_{T} T .
\end{aligned}
$$

The biological meaning of all positive parameters and symbols in the deterministic TB model (1) are listed in Table 1. According to the theory in [17], the deterministic TB model (1) has the following properties:

The positive invariant set $\Gamma$ is given by

$$
\Gamma=\left\{\left(B, M_{u}, M_{i}, T\right) \in \mathbb{R}_{+}^{4}: B(t)<\mathscr{B},\left(M_{u}+M_{i}\right)(t) \leq \frac{s_{M}}{\min \left\{\mu_{M}, b\right\}}, T(t)<\frac{1}{\mu_{T}}\left(s_{T}+\frac{c_{M}}{e_{M}} \mathscr{M}+\frac{c_{B}}{e_{B}} \mathscr{B}\right), \quad \text { where } \mathscr{M}:=\frac{s_{M}}{\min \left\{\mu_{M}, b\right\}}\right\} .
$$

The disease-free equilibrium is given by $E_{0}=\left(M_{u_{0}}, T_{0}, B_{0}, M_{i_{0}}\right)=\left(\left(s_{M} / \mu_{M}\right),\left(s_{T} / \mu_{T}\right), 0,0\right)$.

If $\mathscr{R}_{0} \leq 1$, then the disease-free equilibrium is globally asymptotically stable, where $\mathscr{R}_{0}=\delta \mu_{M} / 2\left(\eta_{3} \beta+\right.$ $\left.\left.\zeta) s_{M}+(1 / 2) \sqrt{\left(4 \beta\left(c \mu_{T}+s_{T}\right)\left(\eta_{1} b+\left(\eta_{2} \gamma s_{T} /\right.\right.\right.} \mu_{T} c+s_{T}\right)\right) /$ $\left.\left(\eta_{3} \beta+\zeta\right)\left(b c \mu_{T}+b s_{T}+\gamma s_{T}\right)\right)+\left(\delta^{2} \mu_{M}^{2} /\left(\eta_{3} \beta+\zeta\right)^{2} s_{M}^{2}\right)$.
The tuberculosis model (1) has a unique positive infected equilibrium $E_{1}$ if and only if $\mathscr{R}_{0}>1$ and it is globally asymptotically stable when it exists. The positive infected equilibrium $E_{1}=\left(M_{u_{1}}, T_{1}, B_{1}, M_{i_{1}}\right)$, where $M_{u_{1}}=\left(s_{M} / \beta B_{1}+\mu_{M}\right), M_{i_{1}}=\left(\beta s_{M} B_{1}\left(T_{1}+c\right) /\right.$ $\left.\left(\beta B_{1}+\mu_{M}\right)\left(b c+b T_{1}+\gamma T_{1}\right)\right)$, and

$$
T_{1}=\frac{-c\left(\left(\left(\beta B_{1}+\mu_{M}\right) \delta+\left(\left(\eta_{1}-\eta_{3}\right) \beta-\zeta\right) s_{M}\right) K-\delta B_{1}\left(\beta B_{1}+\mu_{M}\right)\right) b}{\left.\left((\gamma+b)\left(\beta B_{1}+\mu_{M}\right) \delta+\left(\left(\left(\eta_{1}-\eta_{3}\right) b+\gamma\left(\eta_{2}-\eta_{3}\right)\right) \beta-\zeta(\gamma+b)\right) s_{M}\right)\right) K-\delta B_{1}(\gamma+b)\left(\beta B_{1}+\mu_{M}\right)},
$$

and $B_{1}$ is determined by

$$
f\left(B_{1}\right)=\left.\frac{\mathrm{d} T}{\mathrm{~d} t}\right|_{M_{u_{1}} T_{1}, M_{i_{1}}}=\left(c_{B} T_{1}\left(e_{M} T_{1}+1\right)\right) B_{1}+\left(-e_{M} \mu_{T} T_{1}^{2}+\left(c_{M} M_{i_{1}}+e_{M} s_{T}-\mu_{T}\right) T_{1}+s_{T}\right)\left(e_{B} T_{1}+1\right)=0
$$

In reality, the environmental noise perverts through and medal with the population dynamics, ecology, environmental sciences, and mathematical biology. Stochasticity has its impact upon various biological [18-25] and other models [26-30]. Many researchers probed stochastic tuberculosis models in which the total host population can be divided into three, four, and five epidemiological classes, respectively, such as susceptible $(S)$, exposed $(E)$, and infected $(I)[31]$; susceptible $(S)$, latent $(L)$, infectious $(I)$, and treated $(T)$ [32]; susceptible $(S)$, exposed $(E)$, infected $(I)$, and recovered $(R)[33]$; and susceptible $(S)$, vaccinated $(V)$, infected with TB in latent stage $(L)$, infected with TB in active stage $(I)$, and treated individuals infected with TB $(T)$ $[34,35]$. On the other hand, Khalid Hattaf et al. $[36,37]$ 
TABLE 1: Biological meaning of variables and parameters of the deterministic model (1).

\begin{tabular}{lc}
\hline Symbol & Interpretation \\
\hline Variables & \\
$M_{u}(t)$ & Mycobacterium tuberculosis (MTB) population at time $t$ \\
$M_{i}(t)$ & Population of uninfected macrophages at time $t$ \\
$T(t)$ & Population of infected macrophages at time $t$ \\
Parameters & Population of immune response CD4+ T cells at time $t$ \\
$\delta$ & Bacterium growth rate \\
$K$ & Carrying capacity \\
$\eta_{1}$ & Death rate of infected macrophages \\
$\eta_{2}$ & Extracellular bacteria $(B)$ rate by burst \\
$\eta_{3}$ & Engulfment rate of uninfected macrophages \\
$c$ & Half saturation rate in uninfected macrophages \\
$\zeta$ & Killing rate of extracellular bacteria \\
$s_{M}$ & Influx rate of uninfected macrophages \\
$\mu_{M}$ & Death rates of uninfected macrophages \\
$\beta$ & Infection rates of uninfected macrophages \\
$b$ & Killing rate of infected macrophages \\
$\gamma$ & Cell-mediated adaptive immune response rate \\
$s_{T}$ & Natural recruitment rate of activated CD4+ T cells \\
$c_{B}$ & Maximum killing rates of infected macrophages \\
$c_{M}$ & Maximum killing rates of extracellular bacteria macrophages \\
$e_{B}$ & Saturating factor of infected macrophages \\
$e_{M}$ & Death rate of activated CD4+ T cells \\
$\mu_{T}$ & Saturating factor of extracellular bacteria macrophages
\end{tabular}

investigated specific functional response and temporary immunity and delayed viral infection models with adaptive immune response like cytotoxic T lymphocyte (CTL) cells which is in MHC class I molecules. In all the above models such as SEI, SLIT, SEIR, SVLIT, the researchers probed on host population, temporary immunity, and CTL cells. However, the authors have not examined the functions of macrophages and immune response CD4+ T cells which are in MHC class II molecules so far.

The macrophages inundate the MTB and quarantine them in a cellular compartment where the bacteria are killed or cannot thrive. If the number of MTB is higher than that of macrophages, it will result in the death of the macrophages in the granuloma. Then the granuloma's core liquidizes and is transmitted through airways to other people. During infection, the MTB cells set sporadically in the alveoli of an individual. The immunologic effects of both infected and uninfected macrophages vary randomly due to environmental sources of MTB. The adaptive immune responses in an individual start with very few number of cells and later vary greatly among individual immune systems. This variation is random, and on the other hand, individuals with smoking habit, weak immunity, malnutrition, etc., possess low immune response and have to be treated with more dosages of antigens to increase the immunity contingently.

This study is challenging and useful in the sense that TB in its various dimensions and nature can be disclosed. Based on the above factors, we put forth the stochastic hostpathogen TB model with adaptive immune response. The stochastic host-pathogen TB model on the basis of the influence of the environment on the Mycobacterium tuberculosis and macrophage population was manifested by stochastic perturbations and it is proportional to each state $[38,39]$.

We establish the following stochastic host-pathogen TB model:

$$
\begin{aligned}
\mathrm{d} B(t) & =\left[\delta B\left(1-\frac{B}{K}\right)+M_{i}\left(\eta_{1} b+\eta_{2} \gamma \frac{T}{c+T}\right)-M_{u} B\left(\zeta+\eta_{3} \beta\right)\right] \mathrm{d} t+\sigma_{1} B \mathrm{~d} W_{1}(t), \\
\mathrm{d} M_{u}(t) & =\left[s_{M}-\mu_{M} M_{u}-\beta M_{u} B\right] \mathrm{d} t+\sigma_{2} M_{u} \mathrm{~d} W_{2}(t), \\
\mathrm{d} M_{i}(t) & =\left[\beta M_{u} B-b M_{i}-\gamma M_{i} \frac{T}{c+T}\right] \mathrm{d} t+\sigma_{3} M_{i} \mathrm{~d} W_{3}(t), \\
\mathrm{d} T(t) & =\left[s_{T}+c_{M} M_{i} \frac{T}{1+e_{m} T}+c_{B} B \frac{T}{1+e_{B} T}-\mu_{T} T\right] \mathrm{d} t+\sigma_{4} T \mathrm{~d} W_{4}(t) .
\end{aligned}
$$


Let $W(t)=\left(W_{1}(t), W_{2}(t), W_{3}(t), W_{4}(t)\right)$ be a 4 -dimensional Wiener process defined on the given probability space. The components of $W(t)$ are supposed to be mutually independent. In the stochastic model (5), the nonnegative constants $\sigma_{1}, \sigma_{2}, \sigma_{3}$, and $\sigma_{4}$ denote the intensities of the environmental white noise.

The intention of this literature is structured as follows. In Section 2, the existence of the global and positive solutions to the stochastic host-pathogen TB model (5) is examined. In Section 3, sufficient conditions for the extinction of the tubercle bacillus are probed. In Section 4, we probe that there is a unique ergodic stationary distribution of the positive solutions of the stochastic TB model (5) under some conditions. In Section 5, we demonstrate some numerical simulations that validate our analytical findings. Eventually, Section 6 provides conclusions and future directions.

\section{Global Positive Solutions of the Stochastic TB Model}

Since $B, M_{u}, M_{i}$, and $T$ in the stochastic TB system (5) denotes the population of MTB, uninfected macrophages, infected macrophages, and immune response CD4+ T cells, respectively, they should be nonnegative. For a stochastic differential equation to have a unique global (i.e., no explosion in a finite time) solution for any given initial value, the coefficients of the equation are eventually required to satisfy the linear growth condition and local Lipschitz condition. Yet, the coefficients of equation (5) do not satisfy the linear growth condition; nevertheless, they are locally Lipschitz continuous, and thus, the solution of equation (5) may explode at a finite time. Hence, for further studies, we should set some preconditions under which stochastic system (5) has unique global positive solutions.

Theorem 1. For any given initial value $\left(B(0), M_{u}(0)\right.$, $\left.M_{i}(0), T(0)\right) \in \mathbb{R}_{+}^{4}$, there exists unique positive solutions $\left(B(t), M_{u}(t), M_{i}(t), T(t)\right)$ of the stochastic TB model (5) on $t \geq 0$ and the solution will remain in $\mathbb{R}_{+}^{4}$ with probability one.

Proof. It is clear that the stochastic TB system (5) satisfies the local Lipschitz condition for any given initial value $\left(B(0), M_{u}(0), M_{i}(0), T(0)\right) \in \mathbb{R}_{+}^{4}$. Then, there exist unique maximum local solutions $\left(B(t), M_{u}(t), M_{i}(t), T(t)\right)$ on $t \in\left[0, \tau_{*}\right)$, where $\tau_{*}$ is the explosion time [43]. To exhibit that the solution is universal, i.e., inevitable to affirm that $\tau_{*}=\infty$ a.s. Basically, we exhibit that $B(t), M_{u}(t), M_{i}(t)$, and $T(t)$ do not explode to infinity in a finite time. Let $\kappa_{0}>0$ be adequately large $\left(B(0), M_{u}(0), M_{i}(0), T(0)\right) \epsilon$ $\left[\left(1 / \kappa_{0}\right), \kappa_{0}\right]$. For each integer $\kappa \geq \kappa_{0}$, define the stopping time [40]:

$$
\tau_{\kappa}=\inf \left\{t \in\left[0, \tau_{*}\right): \min \left\{B(t), M_{u}(t), M_{i}(t), T(t)\right\} \leq \frac{1}{\kappa} \text { or } \max \left\{B(t), M_{u}(t), M_{i}(t), T(t)\right\} \geq \kappa\right\}
$$

with the typical format $\inf \phi=\infty$, where $\phi$ reflects the empty set. Evidently, $\tau_{\kappa}$ is increasing as $\kappa \longrightarrow \infty$. We have $\tau_{\infty}=\lim _{\kappa \longrightarrow \infty} \tau_{\kappa}$; therefore, $\tau_{\infty} \leq \tau_{*}$ a.s. If we evince that $\tau_{\infty}=$ $\infty$ a.s., then $\tau_{*}=\infty$ and $\left(B(t), M_{u}(t), M_{i}(t), T(t)\right) \in \mathbb{R}_{+}^{4}$ a.s. for all $t \geq 0$. Presume that $\tau_{\infty}<\infty$, then there is a pair of constants $\widetilde{T}>0$ and $\varepsilon \in(0,1)$ such that

$$
\mathbb{P}\left\{\tau_{\infty} \leq \widetilde{T}\right\}>\varepsilon
$$

Thus, there exists an integer $\kappa_{1} \geq \kappa_{0}$ such that

$$
\mathbb{P}\left\{\tau_{\kappa} \leq \widetilde{T}\right\} \geq \varepsilon, \quad \text { for all } \kappa \geq \kappa_{1} .
$$

Define a $\mathscr{C}^{2}$-function $V: \mathbb{R}_{+}^{4} \longrightarrow \mathbb{R}_{+}$by

$$
V\left(B, M_{u}, M_{i}, T\right)=\varphi_{2}\left(B-\varphi_{1}-\varphi_{1} \ln \frac{B}{\varphi_{1}}\right)+\left(M_{u}-1-\ln M_{u}\right)+\left(M_{i}-1-\ln M_{i}\right)+\varphi_{2}(T-1-\ln T)
$$

where $\varphi_{1}>0$ and $\varphi_{2}>0$ will be determined later. The nonnegativity of $V\left(B, M_{\mathcal{u}}, M_{i}, T\right)$ function occurs from $u-1-\ln u \geq 0, \forall u>0$.

By Itô's formula to $V$, we obtain

$$
\begin{aligned}
\mathrm{d} V= & \mathscr{L} V \mathrm{~d} t+\varphi_{2} \sigma_{1}\left(B-\varphi_{1}\right) \mathrm{d} W_{1}+\sigma_{2}\left(M_{u}-1\right) \mathrm{d} W_{2} \\
& +\sigma_{3}\left(M_{i}-1\right) \mathrm{d} W_{3}+\varphi_{2} \sigma_{4}(T-1) \mathrm{d} W_{4},
\end{aligned}
$$

where $\mathscr{L} V: \mathbb{R}_{+}^{4} \longrightarrow \mathbb{R}_{+}$is defined as 


$$
\begin{aligned}
& \mathscr{L} V=\varphi_{2}\left(1-\frac{\varphi_{1}}{B}\right)\left[\delta B\left(1-\frac{B}{K}\right)+M_{i}\left(\eta_{1} b+\eta_{2} \gamma \frac{T}{c+T}\right)-M_{u} B\left(\zeta+\eta_{3} \beta\right)\right]+\frac{\varphi_{1} \varphi_{2} \sigma_{1}^{2}}{2} \\
& +\left(1-\frac{1}{M_{u}}\right)\left[s_{M}-\mu_{M} M_{u}-\beta M_{u} B\right]+\frac{\sigma_{2}^{2}}{2}+\left(1-\frac{1}{M_{i}}\right)\left[\beta M_{u} B-b M_{i}-\gamma M_{i} \frac{T}{c+T}\right] \\
& +\frac{\sigma_{3}^{2}}{2}+\varphi_{2}\left(1-\frac{1}{T}\right)\left[s_{T}+c_{M} M_{i} \frac{T}{1+e_{m} T}+c_{B} B \frac{T}{1+e_{B} T}-\mu_{T} T\right]+\frac{\varphi_{2} \sigma_{4}^{2}}{2}, \\
& \mathscr{L} V=-\frac{\varphi_{2} \delta}{K} B^{2}+\frac{\varphi_{2} \delta\left(\varphi_{1}+K\right)}{K} B-\varphi_{1} \varphi_{2} \delta+\varphi_{2} M_{i}\left(\eta_{1} b+\eta_{2} \gamma \frac{T}{c+T}\right)-\varphi_{2} M_{u} B\left(\zeta+\eta_{3} \beta\right) \\
& -\frac{\varphi_{1} \varphi_{2} M_{i}}{B}\left(\eta_{1} b+\eta_{2} \gamma \frac{T}{c+T}\right)+\varphi_{1} \varphi_{2} M_{u}\left(\zeta+\eta_{3} \beta\right)+s_{M}-\mu_{M} M_{u}-\frac{s_{M}}{M_{u}}+\mu_{M}+\beta B \\
& -b M_{i}-\gamma M_{i} \frac{T}{c+T}-\frac{1}{M_{i}}\left(\beta M_{u} B\right)+b+\gamma \frac{T}{c+T}+\varphi_{2} s_{T}+\frac{\varphi_{2} c_{M} M_{i} T}{1+e_{m} T}+\frac{\varphi_{2} c_{B} B T}{1+e_{B} T} \\
& -\varphi_{2} \mu_{T} T-\frac{\varphi_{2} s_{T}}{T}-\frac{\varphi_{2} c_{M} M_{i}}{1+e_{M} T}-\frac{\varphi_{2} c_{B} B}{1+e_{B} T}+\varphi_{2} \mu_{T}+\frac{\varphi_{1} \varphi_{2} \sigma_{1}^{2}}{2}+\frac{\sigma_{2}^{2}}{2}+\frac{\sigma_{3}^{2}}{2}+\frac{\varphi_{2} \sigma_{4}^{2}}{2}, \\
& \mathscr{L} V \leq \max _{B \in \mathbb{R}_{+}}\left\{-\frac{\varphi_{2} \delta}{K} B^{2}+\frac{\varphi_{2} \delta\left(\varphi_{1}+K\right)+K \beta+K \varphi_{2} c_{B}}{K} B\right\}+\left(\varphi_{1} \varphi_{2}\left(\zeta+\eta_{3} \beta\right)-\mu_{M}\right) M_{u} \\
& +\left(\varphi_{2}\left(\eta_{1} b+\eta_{2} \gamma+c_{M}\right)-b\right) M_{i}+s_{M}+\mu_{M}+b+\gamma+\varphi_{2} s_{T}+\varphi_{2} \mu_{T}+\frac{\varphi_{1} \varphi_{2} \sigma_{1}^{2}}{2}+\frac{\sigma_{2}^{2}}{2}+\frac{\sigma_{3}^{2}}{2}+\frac{\varphi_{2} \sigma_{4}^{2}}{2} .
\end{aligned}
$$

Choose $\varphi_{2}=\left(b / \eta_{1} b+\eta_{2} \gamma+c_{M}\right)$ such that

$\varphi_{2}\left(\eta_{1} b+\eta_{2} \gamma+c_{M}\right)-b=0$ and let $\varphi_{1}=\left(\mu_{M} / \varphi_{2}\left(\zeta+\eta_{3} \beta\right)\right)$

such that $\varphi_{1} \varphi_{2}\left(\zeta+\eta_{3} \beta\right)-\mu_{M}=0$, and then we have

$$
\mathscr{L} V \leq \max _{B \in \mathbb{R}_{+}}\left\{-\frac{\varphi_{2} \delta}{K} B^{2}+\frac{\varphi_{2} \delta\left(\varphi_{1}+K\right)+K \beta+K \varphi_{2} c_{B}}{K} B\right\}+s_{M}+\mu_{M}+b+\gamma+\varphi_{2} s_{T}+\varphi_{2} \mu_{T}+\frac{\varphi_{1} \varphi_{2} \sigma_{1}^{2}}{2}+\frac{\sigma_{2}^{2}}{2}+\frac{\sigma_{3}^{2}}{2}+\frac{\varphi_{2} \sigma_{4}^{2}}{2}:=\Lambda,
$$

where $\Lambda$ is a positive constant. Thus,

$$
\begin{gathered}
\mathrm{d} V \leq \Lambda \mathrm{d} t+\varphi_{2} \sigma_{1}\left(B-\varphi_{1}\right) \mathrm{d} W_{1}+\sigma_{2}\left(M_{u}-1\right) \mathrm{d} W_{2} \\
+\sigma_{3}\left(M_{i}-1\right) \mathrm{d} W_{3}+\varphi_{2} \sigma_{4}(T-1) \mathrm{d} W_{4} .
\end{gathered}
$$

Integrate both sides of equation (13) from 0 to $\tau_{\kappa} \wedge \widetilde{T}=\min \left\{\tau_{\kappa}, \widetilde{T}\right\}$

$$
\begin{aligned}
\int_{0}^{\tau_{\kappa} \wedge \tilde{T}} \mathrm{~d} V\left(B(\tau), M_{\mathcal{u}}(\tau), M_{i}(\tau), T(\tau)\right) \leq & \int_{0}^{\tau_{\kappa} \wedge \tilde{T}} \Lambda \mathrm{d} t+\int_{0}^{\tau_{\kappa} \wedge \tilde{T}}\left(\varphi_{2} \sigma_{1}\left(B-\varphi_{1}\right) \mathrm{d} W_{1}+\sigma_{2}\left(M_{u}-1\right) \mathrm{d} W_{2}\right. \\
& \left.+\sigma_{3}\left(M_{i}-1\right) \mathrm{d} W_{3}+\varphi_{2} \sigma_{4}(T-1) \mathrm{d} W_{4}\right) .
\end{aligned}
$$


Taking expectations on both sides of (14) yields

$$
\begin{aligned}
& \mathbb{E} V\left(B\left(\tau_{\kappa} \wedge \widetilde{T}\right), M_{u}\left(\tau_{\kappa} \wedge \widetilde{T}\right), M_{i}\left(\tau_{\kappa} \wedge \widetilde{T}\right), T\left(\tau_{\kappa} \wedge \widetilde{T}\right)\right) \leq V\left(B(0), M_{u}(0), M_{i}(0), T(0)\right)+\mathbb{E} \int_{\tau_{\kappa} \wedge T}^{\tau} \Lambda \mathrm{d} \tau, \\
& \mathbb{E} V\left(B\left(\tau_{\kappa} \wedge \widetilde{T}\right), M_{u}\left(\tau_{\kappa} \wedge \widetilde{T}\right), M_{i}\left(\tau_{\kappa} \wedge \widetilde{T}\right), T\left(\tau_{\kappa} \wedge \widetilde{T}\right)\right) \leq V\left(B(0), M_{u}(0), M_{i}(0), T(0)\right)+\Lambda \widetilde{T} .
\end{aligned}
$$

Let $\Omega_{\kappa}=\left\{\tau_{\kappa} \leq \widetilde{T}\right\}$ for all $\kappa \geq \kappa_{1}$, and by (8), then $\mathbb{P}\left(\Omega_{\kappa}\right) \geq \varepsilon$. Notice that for every $\omega \in \Omega_{\kappa}$, there exists at least
$B\left(\tau_{\kappa}, \omega\right)$ or $M_{u}\left(\tau_{\kappa}, \omega\right)$ or $M_{i}\left(\tau_{\kappa}, \omega\right)$ or $T\left(\tau_{\kappa}, \omega\right)$ which is equal to either $\kappa$ or $(1 / \kappa)$.

If $B\left(\tau_{\kappa}, \omega\right)=\kappa$ or $(1 / \kappa)$, then

$$
\begin{aligned}
\left(B\left(\tau_{\kappa}, \omega\right), M_{u}\left(\tau_{\kappa}, \omega\right), M_{i}\left(\tau_{\kappa}, \omega\right), T\left(\tau_{\kappa}, \omega\right)\right) & \geq \varphi_{2}\left[\left(\kappa-\varphi_{1}-\varphi_{1} \ln \frac{\kappa}{\varphi_{1}}\right) \wedge\left(\frac{1}{\kappa}-\varphi_{1}-\varphi_{1} \ln \frac{1}{\varphi_{1} \kappa}\right)\right] \\
& =\varphi_{1} \varphi_{2}\left[\left(\frac{\kappa}{\varphi_{1}}-1-\ln \frac{\kappa}{\varphi_{1}}\right) \wedge\left(\frac{1}{\kappa \varphi_{1}}-1+\ln \varphi_{1} \kappa\right)\right] .
\end{aligned}
$$

Similarly, if $M_{u}\left(\tau_{\kappa}, \omega\right)=\kappa$ or $(1 / \kappa)$, then

$$
\left(B\left(\tau_{\kappa}, \omega\right), M_{u}\left(\tau_{\kappa}, \omega\right), M_{i}\left(\tau_{\kappa}, \omega\right), T\left(\tau_{\kappa}, \omega\right)\right)
$$$$
\geq(\kappa-1-\ln \kappa) \wedge\left(\frac{1}{\kappa}-1+\ln \kappa\right) .
$$

If $M_{i}\left(\tau_{\kappa}, \omega\right)=\kappa$ or $(1 / \kappa)$, then $\left(B\left(\tau_{\kappa}, \omega\right), M_{u}\left(\tau_{\kappa}, \omega\right), M_{i}\left(\tau_{\kappa}, \omega\right), T\left(\tau_{\kappa}, \omega\right)\right)$

$\geq(\kappa-1-\ln \kappa) \wedge\left(\frac{1}{\kappa}-1+\ln \kappa\right)$.

$$
\begin{aligned}
\left(B\left(\tau_{\kappa}, \omega\right), M_{u}\left(\tau_{\kappa}, \omega\right), M_{i}\left(\tau_{\kappa}, \omega\right), T\left(\tau_{\kappa}, \omega\right)\right) \geq & \varphi_{1} \varphi_{2}\left[\left(\frac{\kappa}{\varphi_{1}}-1-\ln \frac{\kappa}{\varphi_{1}}\right) \wedge\left(\frac{1}{\kappa \varphi_{1}}-1+\ln \varphi_{1} \kappa\right)\right] \\
& \wedge(\kappa-1-\ln \kappa) \wedge\left(\frac{1}{\kappa}-1+\ln \kappa\right) \wedge \varphi_{2}(\kappa-1-\ln \kappa) \wedge\left(\frac{1}{\kappa}-1+\ln \kappa\right) .
\end{aligned}
$$

Then, we attain

$$
\begin{aligned}
V\left(B(0), M_{u}(0), M_{i}(0), T(0)\right)+\Lambda \widetilde{T} & \geq \mathbb{E}\left(1_{\Omega \kappa}(\omega) V\left(B\left(\tau_{\kappa} \wedge \widetilde{T}\right), M_{u}\left(\tau_{\kappa} \wedge \widetilde{T}\right), M_{i}\left(\tau_{\kappa} \wedge \widetilde{T}\right), T\left(\tau_{\kappa} \wedge \widetilde{T}\right)\right)\right), \\
& =\mathbb{E}\left(1_{\Omega \kappa}(\omega) V\left(B\left(\tau_{\kappa}, \omega\right), M_{u}\left(\tau_{\kappa}, \omega\right), M_{i}\left(\tau_{\kappa} \wedge \widetilde{T}\right), T\left(\tau_{\kappa}, \omega\right)\right)\right), \\
& \geq \mathbb{E}\left[1_{\Omega \kappa}(\omega) \varphi_{1} \varphi_{2}\left(\left(\frac{\kappa}{\varphi_{1}}-1-\ln \frac{\kappa}{\varphi_{1}}\right) \wedge\left(\frac{1}{\kappa \varphi_{1}}-1+\ln \varphi_{1} \kappa\right)\right) \wedge(\kappa-1-\ln \kappa) \wedge\left(\frac{1}{\kappa}-1+\ln \kappa\right) \wedge \varphi_{2}\left((\kappa-1-\ln \kappa) \wedge\left(\frac{1}{\kappa}-1+\ln \kappa\right)\right)\right], \\
V\left(B(0), M_{u}(0), M_{i}(0), T(0)\right)+\Lambda \widetilde{T} & \geq \varepsilon\left\{\varphi_{1} \varphi_{2}\left(\left(\frac{\kappa}{\varphi_{1}}-1-\ln \frac{\kappa}{\varphi_{1}}\right) \wedge\left(\frac{1}{\kappa \varphi_{1}}-1+\ln \varphi_{1} \kappa\right)\right) \wedge(\kappa-1-\ln \kappa) \wedge\left(\frac{1}{\kappa}-1+\ln \kappa\right) \wedge \varphi_{2}\left((\kappa-1-\ln \kappa) \wedge\left(\frac{1}{\kappa}-1+\ln \kappa\right)\right)\right\},
\end{aligned}
$$


where $1_{\Omega \kappa}$ is the indicator function of $\Omega_{\kappa}(\omega)$. Letting $\kappa \longrightarrow \infty$, we get $\quad \infty>V\left(B(0), M_{u}(0), M_{i}(0)\right.$, $T(0))+\Lambda \widetilde{T}=\infty$. It is a contradiction, and hence, we obtain $\tau_{\infty}=\infty$ a.s., which completes the proof of Theorem 1 .

Remark 1. Considering the region $\mathbb{R}_{+}^{4}$ is positively invariant, the unique solution of the stochastic TB model (5) exists for any given initial value in $\mathbb{R}_{+}^{4}$. Hence, it is sufficient to probe its dynamics of the host-pathogen stochastic TB model in the region $\mathbb{R}_{+}^{4}$.

Remark 2. As stated in the introduction, immunotherapy with LTBI and active TB individuals and adaptive immune responses are known to be quite effective against MTB. However, the prevailing opinion among public health and medical practitioners in the entire world, prior to 1944 [44], was a less effective treatment for TB patients. During the years between 2016 and 2035, the efforts at the global, regional, and national levels to ease and eradicate the burden of TB disease have the colossus objective "ceasing TB epidemic," within the purview of the UN's Agenda for Sustainable Development and based on the WHO's End TB Strategy [1]. Hence, it is fruitful to study the eradication of the MTB among LTBI and active TB individuals. This is done as follows.

\section{Extinction of the MTB}

In this section, the extinction of the MTB is discussed. The death rate of uninfected macrophages and the intensities of the white noise in the stochastic system (5) are the consequences of the random fluctuations. The population dynamics show direct results on the qualitative outcome of the MTB dynamics in the system with regard to the factors that decide MTB eradication during the long course of time. Firstly, we shall present a lemma which will be used in our analysis.

Lemma 1. Let $\left(B(t), M_{u}(t), M_{i}(t), T(t)\right)$ be the solution of the stochastic $T B$ system (5) with any positive initial value $\left(B(0), M_{u}(0), M_{i}(0), T(0)\right) \in \mathbb{R}_{+}^{4}$. Then

$$
\begin{aligned}
& \lim _{t \rightarrow \infty} \frac{B(t)}{t}=0, \\
& \lim _{t \rightarrow \infty} \frac{M_{u}(t)}{t}=0, \\
& \lim _{t \rightarrow \infty} \frac{M_{i}(t)}{t}=0, \\
& \lim _{t \rightarrow \infty} \frac{T(t)}{t}=0 \text { a.s, }
\end{aligned}
$$

Moreover, if $\mu_{M}>\left(\left(\sigma_{1}^{2} \vee \sigma_{2}^{2} \vee \sigma_{3}^{2} \vee \sigma_{4}^{2}\right) / 2\right)$, then

$$
\begin{aligned}
& \lim _{t \rightarrow \infty} \frac{\int_{0}^{t} B(\tau) \mathrm{d} W_{1}(\tau)}{t}=0, \\
& \lim _{t \rightarrow \infty} \frac{\int_{0}^{t} M_{u}(\tau) \mathrm{d} W_{2}(\tau)}{t}=0, \\
& \lim _{t \rightarrow \infty} \frac{\int_{0}^{t} M_{i}(\tau) \mathrm{d} W_{3}(\tau)}{t}=0, \\
& \lim _{t \rightarrow \infty} \frac{\int_{0}^{t} T(\tau) \mathrm{d} W_{4}(\tau)}{t}=0 .
\end{aligned}
$$

Define a parameter as follows:

$$
\widetilde{\mathscr{R}}_{0}=\frac{4\left[\beta s_{M}+\mu_{M}\left(\delta+b N_{1}+\gamma N_{2}\right)\right]}{\mu_{M}\left(\sigma_{1}^{2} \wedge \sigma_{3}^{2}\right)} .
$$

Theorem 2. For any given initial value $\left(B(0), M_{u}(0)\right.$, $\left.M_{i}(0), T(0)\right) \in \mathbb{R}_{+}^{4}$, then the MTB of the stochastic TB system (5) will cease out if $\mu_{M}>\left(\left(\sigma_{1}^{2} \vee \sigma_{3}^{2}\right) / 2\right)$ and $\widetilde{R}_{0}<1$, i.e., $\lim _{t \longrightarrow \infty} B(t)=0, \lim _{t \longrightarrow \infty} M_{i}(t)=0$ a.s.

Proof. Consider the second and third equations of the stochastic TB system (5). Integrating these equations from 0 to $t$ and dividing both sides by $t$, we get

$$
\begin{aligned}
& \frac{M_{u}(t)-M_{u}(0)}{t}+\frac{M_{i}(t)-M_{i}(0)}{t}=s_{M}-\mu_{M}\left\langle M_{u}\right\rangle \\
& -b\left\langle M_{i}\right\rangle-\gamma\left\langle M_{i} \frac{T}{c+T}\right\rangle+\frac{\sigma_{2}}{t} \int_{0}^{t} M_{u}(\tau) \mathrm{d} W_{2}(\tau) \\
& +\frac{\sigma_{3}}{t} \int_{0}^{t} M_{i}(\tau) \mathrm{d} W_{3}(\tau) .
\end{aligned}
$$

It follows that

$$
\left\langle M_{u}\right\rangle \leq \frac{1}{\mu_{M}}\left[s_{M}-\frac{M_{u}(t)-M_{u}(0)}{t}-\frac{M_{i}(t)-M_{i}(0)}{t}+\frac{\sigma_{2}}{t} \int_{0}^{t} M_{u}(\tau) \mathrm{d} W_{2}(\tau)+\frac{\sigma_{3}}{t} \int_{0}^{t} M_{i}(\tau) \mathrm{d} W_{3}(\tau)\right]
$$


Consequently,

$$
\lim _{t \rightarrow \infty}\left\langle M_{u}\right\rangle \leq \frac{1}{\mu_{M}} \lim _{t \rightarrow \infty}\left[s_{M}-\frac{M_{u}(t)-M_{u}(0)}{t}-\frac{M_{i}(t)-M_{i}(0)}{t}+\frac{\sigma_{2}}{t} \int_{0}^{t} M_{u}(\tau) \mathrm{d} W_{2}(\tau)+\frac{\sigma_{3}}{t} \int_{0}^{t} M_{i}(\tau) \mathrm{d} W_{3}(\tau)\right] .
$$

By Lemma 1, we have

$$
\lim _{t \longrightarrow \infty}\left\langle M_{u}\right\rangle \leq \frac{s_{M}}{\mu_{M}} \text { a.s . }
$$

On the other hand, let $P(t)=B(t)+M_{i}(t)$. Applying Itô's formula, we obtain

$$
\begin{aligned}
\mathrm{d} \ln P(t)= & \frac{1}{B+M_{i}}\left[\delta B\left(1-\frac{B}{K}\right)+M_{i}\left(\eta_{1} b+\eta_{2} \gamma \frac{T}{c+T}\right)-M_{u} B\left(\zeta+\eta_{3} \beta\right)+\beta M_{u} B-b M_{i}\right. \\
& \left.-\gamma M_{i} \frac{T}{c+T}-\frac{1}{2\left(B+M_{i}\right)^{2}}\left(\sigma_{1}^{2} B^{2}+\sigma_{3}^{2} M_{i}^{2}\right)\right] \mathrm{d} t \\
& +\frac{\sigma_{1} B}{B+M_{i}} \mathrm{~d} W_{1}(t)+\frac{\sigma_{3} M_{i}}{B+M_{i}} \mathrm{~d} W_{3}(t) \\
\mathrm{d} \ln P(t) \leq & \frac{1}{B+M_{i}}\left[\delta B+M_{i} b\left(\eta_{1}-1\right)+M_{i} \gamma \frac{T}{c+T}\left(\eta_{2}-1\right)+\beta M_{u} B-\frac{1}{2\left(B+M_{i}\right)^{2}}\left(\sigma_{1}^{2} B^{2}+\sigma_{3}^{2} M_{i}^{2}\right)\right] \mathrm{d} t \\
& +\frac{\sigma_{1} B}{B+M_{i}} \mathrm{~d} W_{1}(t)+\frac{\sigma_{3} M_{i}}{B+M_{i}} \mathrm{~d} W_{3}(t), \\
\leq & {\left[\delta+b N_{1}+\gamma N_{2}+\beta M_{u}-\frac{1}{4}\left(\sigma_{1}^{2} \wedge \sigma_{3}^{2}\right)\right] \mathrm{d} t+\frac{\sigma_{1} B}{B+M_{i}} \mathrm{~d} W_{1}(t)+\frac{\sigma_{3} M_{i}}{B+M_{i}} \mathrm{~d} W_{3}(t) }
\end{aligned}
$$

where $N_{1}=\left(\eta_{1}-1\right)>0$ and $N_{2}=\left(\eta_{2}-1\right)>0$.

$$
\begin{aligned}
\ln \left(B(t)+M_{i}(t)\right) \leq & \ln \left(B(0)+M_{i}(0)\right)+\beta \int_{0}^{t} M_{u}(\tau) \mathrm{d} \tau+\left(\delta+b N_{1}+\gamma N_{2}\right) t-\frac{t}{4}\left(\sigma_{1}^{2} \wedge \sigma_{3}^{2}\right) \\
& +\int_{0}^{t} \frac{\sigma_{1} B}{B+M_{i}} \mathrm{~d} W_{1}(\tau)+\int_{0}^{t} \frac{\sigma_{3} M_{i}}{B+M_{i}} \mathrm{~d} W_{3}(\tau) .
\end{aligned}
$$

Dividing both sides of by $t$ and then taking the limit superior yield

$$
\begin{aligned}
\limsup _{t \rightarrow \infty} \frac{\ln \left(B(t)+M_{i}(t)\right)}{t} & \leq \beta \frac{s_{M}}{\mu_{M}}+\left(\delta+b N_{1}+\gamma N_{2}\right)-\frac{1}{4}\left(\sigma_{1}^{2} \wedge \sigma_{3}^{2}\right) \\
& =\frac{1}{4}\left(\sigma_{1}^{2} \wedge \sigma_{3}^{2}\right)\left(\frac{4\left[\beta s_{M}+\mu_{M}\left(\delta+b N_{1}+\gamma N_{2}\right)\right]}{\mu_{M}\left(\sigma_{1}^{2} \wedge \sigma_{3}^{2}\right)}-1\right), \\
& =\frac{1}{4}\left(\sigma_{1}^{2} \wedge \sigma_{3}^{2}\right)\left(\widetilde{\mathscr{R}}_{0}-1\right)<0 .
\end{aligned}
$$


The conclusion is confirmed.

Remark 3. The immunomodulatory agents are compatible to immune responses such as IL- 2 and IL- 4 to effectively active T helper cells. Immunosuppressive agents such as TNF- $\alpha$ moderate dangerous inflammation and cytokine therapy INF- $\gamma$ tends to accelerate the mycobacterial activity of effector immune cells [42]. All these facts exist in reality, which wipe out the MTB.

Remark 4. Patients have lack of information, lack of money for treatment, side effects, lack of commitment to a long course, social barriers, irregular treatment and do not take proper drugs and so they have a low level of immunity. These are the factors for the TB persistent and prevalence among population.

\section{Ergodic Stationary Distribution}

When considering epidemic dynamical systems, we are interested in when the disease will persist and prevail in a population. In this section, we present some theories about the stationary distribution (see Has'minskii [43]), and we show that there exists an ergodic stationary distribution, which reveals that the disease will persist. Let $X(t)$ be a homogeneous Markov process in $\mathbb{R}_{+}^{4}$, which is described by the following stochastic differential equation:

$$
\mathrm{d} X(t)=f(X(t)) \mathrm{d} t+\sum_{r=1}^{k} g_{r}(X) \mathrm{d} W_{r}(t)
$$

The diffusion matrix is defined as follows:

$$
A(x)=\left(a_{i j}(x)\right), \quad a_{i j}(x)=\sum_{r=1}^{k} g_{r}^{i}(x) g_{r}^{j}(x) .
$$

Lemma 2 (see [39]). The Markov process $X(t)$ has a unique ergodic stationary distribution $\pi($.$) if there exists a bounded$ domain $D \subset \mathbb{R}_{+}^{4}$ with regular boundary $\Gamma$, having the following properties:

$A_{1}$ : there is a positive number $M$ such that $\sum_{i, j=1}^{4} a_{i j}(x) \xi_{i} \xi_{j} \geq \quad M|\xi|^{2}$, for $x \in D, \xi=\left(\xi_{1}, \xi_{2}, \quad \xi_{3}\right.$, $\left.\xi_{4}\right) \in \mathbb{R}_{+}^{4}$.

$A_{2}$ : there exist a nonnegative $\mathscr{C}^{2}$-function $V(x)$ and $a$ positive constant $\widehat{C}$ such that $\mathscr{L} V \leq-\widehat{C}$ for any $x \in \mathbb{R}_{+}^{4} \backslash D$. Then

$$
\mathbb{P}\left\{\lim _{T \longrightarrow \infty} \frac{1}{T} \int_{0}^{T} f(X(t)) \mathrm{d} t=\int_{\mathbb{R}_{+}^{4}} f(x) \pi(\mathrm{d} x)\right\}=1,
$$

for all $x \in \mathbb{R}_{+}^{4}$, where $f($.$) is a function integrable with respect$ to the measure $\pi$.

Define a parameter

$$
\begin{aligned}
& \qquad \mathscr{R}_{0}^{s}=\frac{s_{M} \beta}{\left(\vartheta+\left(\sigma_{1}^{2} / 2\right)\right)\left(\mu_{M}+\left(\sigma_{2}^{2} / 2\right)\right)\left(b+\gamma+\left(\sigma_{3}^{2} / 2\right)\right)}\left(\eta_{1} b+\frac{\eta_{2} \gamma s_{T}}{s_{T}+c \mu_{T}+\left(c \sigma_{4}^{2} / 2\right)}\right), \\
& \text { where } \vartheta=\left(\frac{\zeta+\eta_{3} \beta}{\mu_{M}}\right) s_{M}+\left(\zeta+\eta_{3} \beta\right) M_{u_{1}}+\left(\frac{\zeta+\eta_{3} \beta}{\mu_{M}}\right) \frac{M_{u_{1}} \sigma_{2}^{2}}{2} .
\end{aligned}
$$

Theorem 3. Assume that $\mathscr{R}_{0}^{s}>1$, then for any initial value $\left(B(0), M_{u}(0), M_{i}(0), T(0)\right) \in \mathbb{R}_{+}^{4}$, the stochastic $T B$ system (5) admits a unique stationary distribution $\pi$ (.) and it has the ergodic property.

Proof. The diffusion matrix of the stochastic TB system (5) is given by

$$
\left(\begin{array}{cccc}
\sigma_{1}^{2} B^{2} & 0 & 0 & 0 \\
0 & \sigma_{2}^{2} M_{u}^{2} & 0 & 0 \\
0 & 0 & \sigma_{3}^{2} M_{i}^{2} & 0 \\
0 & 0 & 0 & \sigma_{4}^{2} T^{2}
\end{array}\right)
$$

Select $\tilde{M}=\min \left(B, M_{u}, M_{i}, T\right) \in \tilde{D}_{K} \subset \quad \mathbb{R}_{+}^{4}\left\{\sigma_{1}^{2} B^{2}, \sigma_{2}^{2} M_{u}^{2}, \sigma_{3}^{2} M_{i}^{2}\right.$, $\left.\sigma_{4}^{2} T^{2}\right\}$, and we have

$$
\begin{aligned}
& \sum_{i, j=1}^{4} a_{i j}\left(B, M_{u}, M_{i}, T\right) \xi_{i} \xi_{j}=\left(\begin{array}{llll}
\sigma_{1} B \xi_{1} & \sigma_{2} M_{u} \xi_{2} & \sigma_{3} M_{i} \xi_{3} & \sigma_{4} T \xi_{4}
\end{array}\right)\left(\begin{array}{c}
\sigma_{1} B \xi_{1} \\
\sigma_{2} M_{u} \xi_{2} \\
\sigma_{3} M_{i} \xi_{3} \\
\sigma_{4} T \xi_{4}
\end{array}\right) \\
& =\left(\sigma_{1} B\right)^{2} \xi_{1}^{2}+\left(\sigma_{2} M_{u}\right)^{2} \xi_{2}^{2}+\left(\sigma_{3} M_{i}\right)^{2} \xi_{3}^{2}+\left(\sigma_{4} T\right)^{2} \xi_{4}^{2}, \\
& \geq \tilde{M}\|\xi\|^{2} \quad \text { for } \operatorname{any}\left(B, M_{u}, M_{i}, T\right) \in \widetilde{D}_{k}, \xi=\left(\xi_{1}, \xi_{2}, \xi_{3}, \xi_{4}\right) \in \mathbb{R}^{4} \text {, }
\end{aligned}
$$


where $\quad \widetilde{D}_{k}=[(1 / k), k] \times[(1 / k), k] \times[(1 / k), k] \times[(1 / k), k]$ and $k>1$ is an adequately larger integer, and then condition $A_{1}$ in Lemma 2 holds.

Next, we tend to prove the condition $A_{2}$. Define

$$
V_{1}\left(B, M_{u}, M_{i}\right)=-\ln B-k_{1} \ln M_{u}-k_{2} \ln M_{i},
$$

where $k_{1}$ and $k_{2}$ are positive constants to be resolved later. By applying Itô's formula to $V_{1}$, we get

$$
\begin{aligned}
\mathscr{L} V_{1}= & \frac{-1}{B}\left(\delta B\left(1-\frac{B}{K}\right)+M_{i}\left(\eta_{1} b+\eta_{2} \gamma \frac{T}{c+T}\right)-M_{u} B\left(\zeta+\eta_{3} \beta\right)\right)+\frac{\sigma_{1}^{2}}{2} \\
& -\frac{k_{1}}{M_{u}}\left(s_{M}-\mu_{M} M_{u}-\beta M_{u} B\right)+k_{1} \frac{\sigma_{2}^{2}}{2}-\frac{k_{2}}{M_{i}}\left(\beta M_{u} B-b M_{i}-\gamma M_{i} \frac{T}{c+T}\right)+k_{2} \frac{\sigma_{3}^{2}}{2}, \\
= & \frac{\delta B}{K}-\frac{M_{i} \eta_{1} b}{B}-\frac{M_{i} \eta_{2} \gamma}{B} \frac{T}{c+T}+M_{u}\left(\zeta+\eta_{3} \beta\right)-\delta+\frac{\sigma_{1}^{2}}{2}-k_{1} \frac{s_{M}}{M_{u}}+k_{1} \mu_{M}+k_{1} B \beta+k_{1} \frac{\sigma_{2}^{2}}{2} \\
& -k_{2} \frac{\beta M_{u} B}{M_{i}}+k_{2} b+k_{2} \gamma \frac{T}{c+T}+k_{2} \frac{\sigma_{3}^{2}}{2}, \\
= & -\frac{M_{i} \eta_{1} b}{B}-k_{1} \frac{s_{M}}{M_{u}}-k_{2} \frac{\beta M_{u} B}{M_{i}}+\frac{\delta B}{K}-\frac{M_{i} \eta_{2} \gamma}{B} \frac{T}{c+T}+M_{u}\left(\zeta+\eta_{3} \beta\right)-\delta+\frac{\sigma_{1}^{2}}{2}+k_{1} B \beta \\
& +k_{1}\left(\mu_{M}+\frac{\sigma_{2}^{2}}{2}\right)+k_{2} b+k_{2} \gamma \frac{T}{c+T}+k_{2} \frac{\sigma_{3}^{2}}{2}, \\
\mathscr{L} V_{1} \leq & -3 \sqrt[3]{\eta_{1} b s_{M} \beta k_{1} k_{2}}+\frac{\delta B}{K}-\frac{M_{i} \eta_{2} \gamma}{B} \frac{T}{c+T}+M_{u}\left(\zeta+\eta_{3} \beta\right)+\frac{\sigma_{1}^{2}}{2}+k_{1} B \beta+k_{1}\left(\mu_{M}+\frac{\sigma_{2}^{2}}{2}\right) \\
& +k_{2}\left(b+\gamma+\frac{\sigma_{3}^{2}}{2}\right)-\delta .
\end{aligned}
$$

Choose

Then

$$
\begin{aligned}
& k_{1}=\frac{\eta_{1} b s_{M} \beta}{\left(\mu_{M}+\left(\sigma_{2}^{2} / 2\right)\right)^{2}\left(b+\gamma+\left(\sigma_{3}^{2} / 2\right)\right)}, \\
& k_{2}=\frac{\eta_{1} b s_{M} \beta}{\left(\mu_{M}+\left(\sigma_{2}^{2} / 2\right)\right)\left(b+\gamma+\left(\sigma_{3}^{2} / 2\right)\right)^{2}} .
\end{aligned}
$$

$$
\mathscr{L} V_{1} \leq-\frac{\eta_{1} b s_{M} \beta}{\left(\mu_{M}+\left(\sigma_{2}^{2} / 2\right)\right)\left(b+\gamma+\left(\sigma_{3}^{2} / 2\right)\right)}+\frac{\delta B}{K}-\frac{M_{i} \eta_{2} \gamma}{B} \frac{T}{c+T}+M_{u}\left(\zeta+\eta_{3} \beta\right)+\frac{\sigma_{1}^{2}}{2}+k_{1} B \beta-\delta
$$

Define

$$
V_{2}\left(B, M_{u}, M_{i}, T\right)=V_{1}\left(B, M_{u}, M_{i}\right)-k_{3} c \ln T-k_{4} \ln M_{u}-k_{5} \ln M_{i},
$$


where $k_{3}, k_{4}$, and $k_{5}$ are positive constants to be determined later. By calculating,

$$
\begin{aligned}
\mathscr{L} V_{2} \leq & -\frac{M_{i} \eta_{2} \gamma}{B} \frac{T}{c+T}-\frac{\eta_{1} b s_{M} \beta}{\left(\mu_{M}+\left(\sigma_{2}^{2} / 2\right)\right)\left(b+\gamma+\left(\sigma_{3}^{2} / 2\right)\right)}+\frac{\delta B}{K}+M_{u}\left(\zeta+\eta_{3} \beta\right)+\frac{\sigma_{1}^{2}}{2}+k_{1} B \beta-\delta \\
& -k_{3} \frac{c}{T}\left(s_{T}+c_{M} M_{i} \frac{T}{1+e_{M} T}+c_{B} B \frac{T}{1+e_{B} T}-\mu_{T} T\right)+k_{3} \frac{c \sigma_{4}^{2}}{2}-\frac{k_{4}}{M_{u}}\left(s_{M}-\mu_{M} M_{u}-\beta M_{u} B\right)+k_{4} \frac{\sigma_{2}^{2}}{2} \\
& -\frac{k_{5}}{M_{i}}\left(\beta M_{u} B-b M_{i}-\gamma M_{i} \frac{T}{c+T}\right)+k_{5} \frac{\sigma_{3}^{2}}{2} \\
\leq & -\frac{M_{i} \eta_{2} \gamma}{B} \frac{T}{c+T}-k_{3} \frac{c+T}{T} s_{T}-k_{4} \frac{s_{M}}{M_{u}}-k_{5} \frac{\beta M_{u} B}{M_{i}}-\frac{\eta_{1} b s_{M} \beta}{\left(\mu_{M}+\left(\sigma_{2}^{2} / 2\right)\right)\left(b+\gamma+\left(\sigma_{3}^{2} / 2\right)\right)}+\frac{\delta B}{K} \\
& +M_{u}\left(\zeta+\eta_{3} \beta\right)+\frac{\sigma_{1}^{2}}{2}+k_{1} B \beta-\delta+k_{3} s_{T}-k_{3} c c_{M} M_{i} \frac{1}{1+e_{M} T}-k_{3} c c_{B} B \frac{1}{1+e_{B} T}+k_{3} c \mu_{T} \\
& +k_{3} \frac{c \sigma_{4}^{2}}{2}+k_{4} \mu_{M}+k_{4} B \beta+k_{4} \frac{\sigma_{2}^{2}}{2}+k_{5} b+k_{5} \gamma \frac{T}{c+T}+k_{5} \frac{\sigma_{3}^{2}}{2}, \\
\mathscr{L} V_{2} \leq & -4 \sqrt[4]{\eta_{2} \gamma s_{T} s_{M} \beta k_{3} k_{4} k_{5}}-\frac{\eta_{1} s_{M} \beta}{\left(\mu_{M}+\left(\sigma_{2}^{2} / 2\right)\right)\left(b+\gamma+\left(\sigma_{3}^{2} / 2\right)\right)}+\frac{\delta B}{K}+M_{u}\left(\zeta+\eta_{3} \beta\right)+\frac{\sigma_{1}^{2}}{2}+k_{1} B \beta \\
& +k_{4} B \beta+k_{3}\left(s_{T}+c \mu_{T}+\frac{c \sigma_{4}^{2}}{2}\right)+k_{4}\left(\mu_{M}+\frac{\sigma_{2}^{2}}{2}\right)+k_{5}\left(b+\gamma+\frac{\sigma_{3}^{2}}{2}\right)-\delta .
\end{aligned}
$$

Choose

Hence,

$$
\begin{aligned}
& k_{3}=\frac{\eta_{2} \gamma s_{T} s_{M} \beta}{\left(s_{T}+c \mu_{T}+\left(c \sigma_{4}^{2} / 2\right)\right)^{2}\left(\mu_{M}+\left(\sigma_{2}^{2} / 2\right)\right)\left(b+\gamma+\left(\sigma_{3}^{2} / 2\right)\right)}, \\
& k_{4}=\frac{\eta_{2} \gamma s_{T} s_{M} \beta}{\left(s_{T}+c \mu_{T}+\left(c \sigma_{4}^{2} / 2\right)\right)\left(\mu_{M}+\left(\sigma_{2}^{2} / 2\right)\right)^{2}\left(b+\gamma+\left(\sigma_{3}^{2} / 2\right)\right)}, \\
& k_{5}=\frac{\eta_{2} \gamma s_{T} s_{M} \beta}{\left(s_{T}+c \mu_{T}+\left(c \sigma_{4}^{2} / 2\right)\right)\left(\mu_{M}+\left(\sigma_{2}^{2} / 2\right)\right)\left(b+\gamma+\left(\sigma_{3}^{2} / 2\right)\right)^{2}} .
\end{aligned}
$$

$$
\begin{aligned}
\mathscr{L} V_{2} \leq & -\frac{\eta_{2} \gamma s_{T} s_{M} \beta}{\left(s_{T}+c \mu_{T}+\left(c \sigma_{4}^{2} / 2\right)\right)\left(\mu_{M}+\left(\sigma_{2}^{2} / 2\right)\right)\left(b+\gamma+\left(\sigma_{3}^{2} / 2\right)\right)}-\frac{\eta_{1} b s_{M} \beta}{\left(\mu_{M}+\left(\sigma_{2}^{2} / 2\right)\right)\left(b+\gamma+\left(\sigma_{3}^{2} / 2\right)\right)} \\
& +M_{u}\left(\zeta+\eta_{3} \beta\right)+\frac{\sigma_{1}^{2}}{2}+\left(\frac{\delta}{K}+k_{1} \beta+k_{4} \beta\right) B-\delta, \\
\mathscr{L} V_{2} \leq & -\frac{s_{M} \beta}{\left(\mu_{M}+\left(\sigma_{2}^{2} / 2\right)\right)\left(b+\gamma+\left(\sigma_{3}^{2} / 2\right)\right)}\left(\eta_{1} b+\frac{\eta_{2} \gamma s_{T}}{s_{T}+c \mu_{T}+\left(c \sigma_{4}^{2} / 2\right)}\right)+M_{u}\left(\zeta+\eta_{3} \beta\right)+\frac{\sigma_{1}^{2}}{2} \\
& +\left(\frac{\delta}{K}+k_{1} \beta+k_{4} \beta\right) B-\delta .
\end{aligned}
$$


Define

$$
\begin{aligned}
U_{1}\left(B, M_{u}, M_{i}, T\right)= & V_{2}\left(B, M_{u}, M_{i}, T\right)+\left(\frac{\zeta+\eta_{3} \beta}{\mu_{M}}\right)\left(M_{u}-M_{u_{1}}-M_{u_{1}} \ln \frac{M_{u}}{M_{u_{1}}}\right), \\
\mathscr{L} U_{1} \leq & -\frac{s_{M} \beta}{\left(\mu_{M}+\left(\sigma_{2}^{2} / 2\right)\right)\left(b+\gamma+\left(\sigma_{3}^{2} / 2\right)\right)}\left(\eta_{1} b+\frac{\eta_{2} \gamma s_{T}}{s_{T}+c \mu_{T}+\left(c \sigma_{4}^{2} / 2\right)}\right)+M_{u}\left(\zeta+\eta_{3} \beta\right)+\frac{\sigma_{1}^{2}}{2} \\
& +\left(\frac{\delta}{K}+k_{1} \beta+k_{4} \beta\right) B-\delta+\left(\frac{\zeta+\eta_{3} \beta}{\mu_{M}}\right)\left(1-\frac{M_{u_{1}}}{M_{u}}\right)\left(s_{M}-\mu_{M} M_{u}-\beta M_{u} B\right)+\left(\frac{\zeta+\eta_{3} \beta}{\mu_{M}}\right) \frac{M_{u_{1}} \sigma_{2}^{2}}{2}, \\
\leq & -\frac{s_{M} \beta}{\left(\mu_{M}+\left(\sigma_{2}^{2} / 2\right)\right)\left(b+\gamma+\left(\sigma_{3}^{2} / 2\right)\right)}\left(\eta_{1} b+\frac{\eta_{2} \gamma s_{T}}{s_{T}+c \mu_{T}+\left(c \sigma_{4}^{2} / 2\right)}\right)+\left(\frac{\zeta+\eta_{3} \beta}{\mu_{M}}\right) s_{M}+\frac{\sigma_{1}^{2}}{2}+\left(\zeta+\eta_{3} \beta\right) M_{u_{1}} \\
& +\left(\frac{\zeta+\eta_{3} \beta}{\mu_{M}}\right) \frac{M_{u_{1}} \sigma_{2}^{2}}{2}+\left(\frac{\delta}{K}+k_{1} \beta+k_{4} \beta++\left(\frac{\zeta+\eta_{3} \beta}{\mu_{M}}\right) \beta M_{u_{1}}\right) B, \\
\mathscr{L} U_{1} \leq & -\frac{s_{M} \beta}{\left(\mu_{M}+\left(\sigma_{2}^{2} / 2\right)\right)\left(b+\gamma+\left(\sigma_{3}^{2} / 2\right)\right)}\left(\eta_{1} b+\frac{\eta_{2} \gamma s_{T}}{s_{T}+c \mu_{T}+\left(c \sigma_{4}^{2} / 2\right)}\right)+\vartheta+\frac{\sigma_{1}^{2}}{2}+\phi B \\
= & -\left(\vartheta+\frac{\sigma_{1}^{2}}{2}\right)\left(\mathscr{R}_{0}^{s}-1\right)+\phi B=-\lambda+\phi B,
\end{aligned}
$$

where

$$
\begin{aligned}
\lambda & :=-\left(\vartheta+\frac{\sigma_{1}^{2}}{2}\right)\left(\mathscr{R}_{0}^{s}-1\right) \\
\mathscr{R}_{0}^{s} & :=\frac{s_{M} \beta}{\left(\vartheta+\left(\sigma_{1}^{2} / 2\right)\right)\left(\mu_{M}+\left(\sigma_{2}^{2} / 2\right)\right)\left(b+\gamma+\left(\sigma_{3}^{2} / 2\right)\right)}\left(\eta_{1} b+\frac{\eta_{2} \gamma s_{T}}{s_{T}+c \mu_{T}+\left(c \sigma_{4}^{2} / 2\right)}\right) \\
\vartheta & :=\left(\frac{\zeta+\eta_{3} \beta}{\mu_{M}}\right) s_{M}+\left(\zeta+\eta_{3} \beta\right) M_{u_{1}}+\left(\frac{\zeta+\eta_{3} \beta}{\mu_{M}}\right) \frac{M_{u_{1}} \sigma_{2}^{2}}{2} \\
\phi & :=\left(\frac{\delta}{K}+k_{1} \beta+k_{4} \beta++\left(\frac{\zeta+\eta_{3} \beta}{\mu_{M}}\right) \beta M_{u_{1}}\right) .
\end{aligned}
$$

Define a $\mathscr{C}^{2}$-function $U: \mathbb{R}^{4} \longrightarrow \mathbb{R}$, as follows:

$U\left(B, M_{u}, M_{i}, T\right)=M U_{1}+U_{2}+U_{3}+U_{4}+U_{5}+U_{6}$,

where $U_{2}=-\ln B, U_{3}=-\ln M_{u}, U_{4}=-\ln M_{i}, U_{5}=-\ln T$, $U_{6}=(1 / \theta+1)\left(\left(B / \eta_{1} b+\eta_{2} \gamma\right)+M_{u}+M_{i}+(T / 4)\right)^{\theta+1}$, in which $\theta$ is a sufficiently small constant satisfying $0<\theta<\min$ $\left\{\left(\mu_{M} / \sigma_{2}^{2}\right),\left(b / 1+\left(c_{M} / 4\right)+\sigma_{3}^{2}\right),\left(T / \sigma_{4}^{2}\right)\right\} ; b>\quad\left(1+\quad\left(c_{M} / 4\right)\right)$, and select a suitable constant $M>0$ which satisfies the following condition:

$$
-M \lambda+E \leq-2
$$


where

$$
\begin{aligned}
E= & \sup _{\left(B, M_{u}, M_{i}, T\right) \in \mathbb{R}_{+}^{4}}\left\{\frac{\delta B}{K}+M_{u}\left(\zeta+\eta_{3} \beta\right)+\beta B-\frac{\delta B^{\theta+2}}{2\left(\eta_{1} b+\eta_{2} \gamma\right) K}-\frac{\mu_{M}}{2}\left(M_{u}\right)^{\theta+1}\right. \\
& \left.-\frac{\left(b-\left(1+c_{M} / 4\right)\right)}{2}\left(M_{i}\right)^{\theta+1}-\frac{\mu_{T}}{8}(T)^{\theta+1}+F-\delta+\mu_{M}+b+\gamma+\mu_{T}+\frac{\sigma_{1}^{2}}{2}+\frac{\sigma_{2}^{2}}{2}+\frac{\sigma_{3}^{2}}{2}+\frac{\sigma_{4}^{2}}{2}\right\} .
\end{aligned}
$$

Easily we can check that

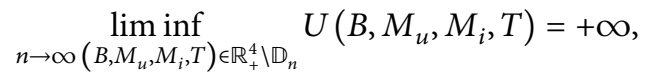

where $\mathbb{D}_{n}=((1 / n), n) \times((1 / n), n) \times((1 / n), n) \times((1 / n), n)$. Furthermore, $U\left(B, M_{u}, M_{i}, T\right)$ is not only continuous.
Hence, $U\left(B, M_{u}, M_{i}, T\right)$ has a minimum point $\left(\widetilde{B_{0}}, \widetilde{M_{u_{0}}}, \widetilde{M_{i_{0}}}, \widetilde{T_{0}}\right)$ in the interior of $\mathbb{R}_{+}^{4}$. Then, we define a $\mathscr{C}^{2}$-function

$\widehat{U}\left(B, M_{\mathcal{u}}, M_{i}, T\right)=U\left(B, M_{\mathcal{u}}, M_{i}, T\right)-\widetilde{U}\left(\widetilde{B_{0}}, \widetilde{M_{u_{0}}}, \widetilde{M_{i_{0}}}, \widetilde{T_{0}}\right)$. By applying Itô's formula, we obtain

$$
\begin{aligned}
& \mathscr{L} U_{2}=-\delta\left(1-\frac{B}{K}\right)-\frac{M_{i}}{B}\left(\eta_{1} b+\eta_{2} \gamma \frac{T}{c+T}\right)+M_{u}\left(\zeta+\eta_{3} \beta\right)+\frac{\sigma_{1}^{2}}{2} \\
& \leq-\delta+\frac{\delta B}{K}+M_{u}\left(\zeta+\eta_{3} \beta\right)+\frac{\sigma_{1}^{2}}{2} \\
& \mathscr{L} U_{3}=-\frac{s_{M}}{M_{u}}+\mu_{M}+\beta B+\frac{\sigma_{2}^{2}}{2} \\
& \mathscr{L} U_{4}=-\frac{\beta M_{u} B}{M_{i}}+b+\gamma \frac{T}{c+T}+\frac{\sigma_{3}^{2}}{2} \leq-\frac{\beta M_{u} B}{M_{i}}+b+\gamma+\frac{\sigma_{3}^{2}}{2}, \\
& \mathscr{L} U_{5}=-\frac{s_{T}}{T}-c_{M} M_{i} \frac{1}{1+e_{M} T}-c_{B} B \frac{1}{1+e_{B} T}+\mu_{T}+\frac{\sigma_{4}^{2}}{2} \\
& \leq-\frac{s_{T}}{T}+\mu_{T}+\frac{\sigma_{4}^{2}}{2} \\
& \mathscr{L} U_{6}=\left(\frac{B}{\eta_{1} b+\eta_{2} \gamma}+M_{u}+M_{i}+\frac{T}{4}\right)^{\theta}\left(\frac{1}{\eta_{1} b+\eta_{2} \gamma}\left(\delta B\left(1-\frac{B}{K}\right)+M_{i}\left(\eta_{1} b+\eta_{2} \gamma \frac{T}{c+T}\right)-M_{u} B\left(\varsigma+\eta_{3} \beta\right)\right)+s_{M}\right. \\
& \left.-\mu_{M} M_{u}-b M_{i}-\gamma M_{i} \frac{T}{c+T}+\frac{s_{T}}{4}+c_{M} M_{i} \frac{T}{4\left(1+e_{M} T\right)}+c_{B} B \frac{T}{4\left(1+e_{B} T\right)}-\frac{\mu_{T} T}{4}\right) \\
& +\frac{\theta}{2}\left(\frac{B}{\eta_{1} b+\eta_{2} \gamma} M_{u}+M_{i}+\frac{T}{4}\right)^{\theta-1} \times\left(\left(\sigma_{1} \frac{B}{\eta_{1} b+\eta_{2} \gamma}\right)^{2}+\sigma_{2}^{2} M_{u}^{2}+\sigma_{3}^{2} M_{i}^{2}+\sigma_{4}^{2}\left(\frac{T}{4}\right)^{2}\right), \\
& \leq\left(\frac{B}{\eta_{1} b+\eta_{2} \gamma}+M_{u}+M_{i}+\frac{T}{4}\right)^{\theta}\left(s_{M}+\frac{s_{T}}{4}+\left(\frac{\delta}{\eta_{1} b+\eta_{2} \gamma}+\frac{c_{B} B}{4}\right) B-\frac{\delta B^{2}}{\left(\eta_{1} b+\eta_{2} \gamma\right) K}-\mu_{M} M_{u}-\left(b-\left(1+\frac{c_{M}}{4}\right)\right) M_{i}-\frac{\mu_{T} T}{4}\right) \\
& +\frac{\theta}{2}\left(\frac{B}{\eta_{1} b+\eta_{2} \gamma} M_{u}+M_{i}+\frac{T}{4}\right)^{\theta-1} \times\left(\sigma_{1}^{2}\left(\frac{B}{\eta_{1} b+\eta_{2} \gamma}\right)^{2}+\sigma_{2}^{2} M_{u}^{2}+\sigma_{3}^{2} M_{i}^{2}+\sigma_{4}^{2}\left(\frac{T}{4}\right)^{2}\right),
\end{aligned}
$$




$$
\begin{aligned}
\leq & \left(s_{M}+\frac{s_{T}}{4}\right)\left(\frac{B}{\eta_{1} b+\eta_{2} \gamma}+M_{u}+M_{i}+\frac{T}{4}\right)^{\theta}+\left(\frac{\delta}{\eta_{1} b+\eta_{2} \gamma}+\frac{c_{B} B}{4}\right) \\
& \times\left(\frac{B}{\eta_{1} b+\eta_{2} \gamma}+M_{u}+M_{i}+\frac{T}{4}\right)^{\theta} B-\frac{\delta B^{\theta+2}}{\left(\eta_{1} b+\eta_{2} \gamma\right) K}-\mu_{M}\left(M_{u}\right)^{\theta+1} \\
& -\left(b-\left(1+\frac{c_{M}}{4}\right)\right)\left(M_{i}\right)^{\theta+1}-\frac{\mu_{T} T}{4}(T)^{\theta+1}+\frac{\theta}{2}\left(\sigma_{1}^{2}\left(\frac{B}{\eta_{1} b+\eta_{2} \gamma}\right)^{\theta+1}+\sigma_{2}^{2} M_{u}^{\theta+1}+\sigma_{3}^{2} M_{i}^{\theta+1}+\sigma_{4}^{2}\left(\frac{T}{4}\right)^{\theta+1}\right) \\
\mathscr{L} U_{6} \leq & -\frac{\delta B^{\theta+2}}{2\left(\eta_{1} b+\eta_{2} \gamma\right) K}-\frac{\mu_{M}}{2}\left(M_{u}\right)^{\theta+1}-\frac{\left(b-\left(1+\left(c_{M} / 4\right)\right)\right)}{2}\left(M_{i}\right)^{\theta+1}-\frac{\mu_{T}}{8}(T)^{\theta+1}+F
\end{aligned}
$$

where

$$
\begin{aligned}
F= & \sup _{\left(B, M_{u}, M_{i}, T\right) \in \mathbb{R}_{+}^{4}}\left\{-\frac{\delta B^{\theta+2}}{2\left(\eta_{1} b+\eta_{2} \gamma\right) K}-\frac{\mu_{M}}{2}\left(M_{u}\right)^{\theta+1}-\frac{\left(b-\left(1+\left(c_{M} / 4\right)\right)\right)}{2}\left(M_{i}\right)^{\theta+1}-\frac{\mu_{T}}{8}(T)^{\theta+1}\right. \\
& +\left(s_{M}+\frac{s_{T}}{4}\right)\left(\frac{B}{\eta_{1} b+\eta_{2} \gamma}+M_{u}+M_{i}+\frac{T}{4}\right)^{\theta}+\left(\frac{\delta}{\eta_{1} b+\eta_{2} \gamma}+\frac{c_{B}}{4}\right) \times\left(\frac{B}{\eta_{1} b+\eta_{2} \gamma}+M_{u}+M_{i}+\frac{T}{4}\right)^{\theta} B \\
& \left.+\frac{\theta}{2}\left(\sigma_{1}^{2}\left(\frac{B}{\eta_{1} b+\eta_{2} \gamma}\right)^{\theta+1}+\sigma_{2}^{2} M_{u}^{\theta+1}+\sigma_{3}^{2} M_{i}^{\theta+1}+\sigma_{4}^{2}\left(\frac{T}{4}\right)^{\theta+1}\right)\right\} .
\end{aligned}
$$

It follows that

$$
\begin{aligned}
\mathscr{L} \widehat{U} \leq & -M \lambda+M \phi B+\frac{\delta B}{K}+M_{u}\left(\zeta+\eta_{3} \beta\right)-\frac{s_{M}}{M_{u}}+\beta B-\frac{\beta M_{u} B}{M_{i}}-\frac{s_{T}}{T}-\frac{\delta B^{\theta+2}}{2\left(\eta_{1} b+\eta_{2} \gamma\right) K} \\
& -\frac{\mu_{M}}{2}\left(M_{u}\right)^{\theta+1}-\frac{\left(b-\left(1+\left(c_{M} / 4\right)\right)\right)}{2}\left(M_{i}\right)^{\theta+1}-\frac{\mu_{T}}{8}(T)^{\theta+1}+F-\delta+\mu_{M}+b+\gamma+\mu_{T}+\frac{\sigma_{1}^{2}}{2}+\frac{\sigma_{2}^{2}}{2}+\frac{\sigma_{3}^{2}}{2}+\frac{\sigma_{4}^{2}}{2} .
\end{aligned}
$$

Now we are in the portion to construct a compact subset $\mathbb{D}_{\epsilon}$ such that condition $A_{2}$ in Lemma 2 holds. Define the following bounded closed set

$$
\mathbb{D}_{\epsilon}=\left\{\left(B, M_{u}, M_{i}, T\right) \in \mathbb{R}_{+}^{4}: \epsilon \leq B \leq \frac{1}{\epsilon}, \epsilon \leq M_{u} \leq \frac{1}{\epsilon}, \epsilon^{3} \leq M_{i} \leq \frac{1}{\epsilon^{3}}, \epsilon \leq T \leq \frac{1}{\epsilon}\right\}
$$

where $0<\epsilon<1$ is a sufficiently small constant. In the set

$$
\begin{array}{r}
-\frac{\beta}{\epsilon}+G \leq-1, \\
-\frac{s_{T}}{\epsilon}+G \leq-1, \\
-\frac{\delta}{4\left(\eta_{1} b+\eta_{2} \gamma\right) K} \frac{1}{\epsilon^{\theta+2}}+H \leq-1,
\end{array}
$$$$
\mathbb{R}_{+}^{4} \backslash \mathbb{D}_{\epsilon} \text {, we can choose } \epsilon \text { sufficiently small such that the }
$$
following conditions hold:

$$
\begin{array}{r}
-M \lambda+M \phi \epsilon+E \leq-1, \\
-\frac{s_{M}}{\epsilon}+G \leq-1,
\end{array}
$$




$$
\begin{array}{r}
-\frac{\mu_{M}}{4} \frac{1}{\epsilon^{\theta+1}}+J \leq-1, \\
-\frac{\left(b-\left(1+\left(c_{M} / 4\right)\right)\right)}{4} \frac{1}{\epsilon^{3 \theta+3}}+N \leq-1, \\
-\frac{\mu_{T}}{16} \frac{1}{\epsilon^{\theta+1}}+O \leq-1,
\end{array}
$$

where $E, G, H, J, N$, and $O$ are positive constants which can be found from the equations (50), (66), (70), (72), (74), and (76). Conveniently, we can divide $\mathbb{R}_{+}^{4} \backslash \mathbb{D}_{\epsilon}$ into the following eight domains:

$$
\begin{aligned}
& \mathbb{D}_{1}=\left\{\left(B, M_{u}, M_{i}, T\right) \in \mathbb{R}_{+}^{4}: 0<B<\epsilon\right\}, \mathbb{D}_{2}=\left\{\left(B, M_{u},\right.\right. \\
& \left.\left.M_{i}, T\right) \in \mathbb{R}_{+}^{4}: 0<M_{u}<\epsilon\right\}, \\
& \mathbb{D}_{3}=\left\{\left(B, M_{u}, M_{i}, T\right) \in \mathbb{R}_{+}^{4}: 0<M_{i}<\epsilon^{3}, B \geq \epsilon, M_{u} \geq \epsilon\right\}, \\
& \mathbb{D}_{4}=\left\{\left(B, M_{u}, M_{i}, T\right) \in \mathbb{R}_{+}^{4}: 0<T<\epsilon\right\}, \\
& \mathbb{D}_{5}=\left\{\left(B, M_{u}, M_{i}, T\right) \in \mathbb{R}_{+}^{4}: B>(1 / \epsilon)\right\}, \\
& \mathbb{D}_{6}=\left\{\left(B, M_{u}, M_{i}, T\right) \in \mathbb{R}_{+}^{4}: M_{u}>(1 / \epsilon)\right\}, \\
& \mathbb{D}_{7}=\left\{\left(B, M_{u}, M_{i}, T\right) \in \mathbb{R}_{+}^{4}: M_{i}>\left(1 / \epsilon^{3}\right)\right\}, \\
& \mathbb{D}_{8}=\left\{\left(B, M_{u}, M_{i}, T\right) \in \mathbb{R}_{+}^{4}: T>(1 / \epsilon)\right\} .
\end{aligned}
$$

Clearly, $\quad \mathbb{D}_{\epsilon}^{c}=\mathbb{D}_{1} \cup \mathbb{D}_{2} \cup \mathbb{D}_{3} \cup \mathbb{D}_{4} \cup \mathbb{D}_{5} \cup \mathbb{D}_{6} \cup \mathbb{D}_{7} \cup \mathbb{D}_{8}$. Next, we prove that $\mathscr{L} \vec{U} \leq-1$ on $\mathbb{D}_{\epsilon}^{c}$, which is equivalent to proving it on the above eight domains, respectively.

Case 1. If $\left(B, M_{\mathcal{u}}, M_{i}, T\right) \in \mathbb{D}_{1}$, we have

$$
\begin{aligned}
\mathscr{L} \widehat{U} \leq & -M \lambda+M \phi B+\frac{\delta B}{K}+M_{u}\left(\zeta+\eta_{3} \beta\right)+\beta B-\frac{\delta B^{\theta+2}}{2\left(\eta_{1} b+\eta_{2} \gamma\right) K}-\frac{\mu_{M}}{2}\left(M_{u}\right)^{\theta+1} \\
& -\frac{\left(b-\left(1+\left(c_{M} / 4\right)\right)\right)}{2}\left(M_{i}\right)^{\theta+1}-\frac{\mu_{T}}{8}(T)^{\theta+1}+F-\delta+\mu_{M}+b+\gamma+\mu_{T}+\frac{\sigma_{1}^{2}}{2}+\frac{\sigma_{2}^{2}}{2}+\frac{\sigma_{3}^{2}}{2}+\frac{\sigma_{4}^{2}}{2}, \\
\leq & -M \lambda+M \phi B+E \leq-M \lambda+M \phi \epsilon+E .
\end{aligned}
$$

According to (56) and (64), we get that $\mathscr{L} \widehat{U} \leq-1$ for any $\left(B, M_{u}, M_{i}, T\right) \in \mathbb{D}_{1}$.

Case 2. If $\left(B, M_{u}, M_{i}, T\right) \in \mathbb{D}_{2}$, we attain

$$
\begin{aligned}
\mathscr{L} \widehat{U} \leq & -\frac{s_{M}}{M_{u}}+M \phi B+\frac{\delta B}{K}+M_{u}\left(\zeta+\eta_{3} \beta\right)+\beta B-\frac{\delta B^{\theta+2}}{2\left(\eta_{1} b+\eta_{2} \gamma\right) K}-\frac{\mu_{M}}{2}\left(M_{u}\right)^{\theta+1} \\
& -\frac{\left(b-\left(1+\left(c_{M} / 4\right)\right)\right)}{2}\left(M_{i}\right)^{\theta+1}-\frac{\mu_{T}}{8}(T)^{\theta+1}+F-\delta+\mu_{M}+b+\gamma+\mu_{T}+\frac{\sigma_{1}^{2}}{2}+\frac{\sigma_{2}^{2}}{2}+\frac{\sigma_{3}^{2}}{2}+\frac{\sigma_{4}^{2}}{2}, \\
\leq & -\frac{s_{M}}{M_{u}}+G \leq-\frac{s_{M}}{\epsilon}+G,
\end{aligned}
$$

where

$$
\begin{aligned}
G= & \sup _{\left(B, M_{u}, M_{i}, T\right) \in \mathbb{R}_{+}^{4}}\left\{M \phi B+\frac{\delta B}{K}+M_{u}\left(\zeta+\eta_{3} \beta\right)+\beta B-\frac{\delta B^{\theta+2}}{2\left(\eta_{1} b+\eta_{2} \gamma\right) K}-\frac{\mu_{M}}{2}\left(M_{u}\right)^{\theta+1}\right. \\
& \left.-\frac{\left(b-\left(1+\left(c_{M} / 4\right)\right)\right)}{2}\left(M_{i}\right)^{\theta+1}-\frac{\mu_{T}}{8}(T)^{\theta+1}+F-\delta+\mu_{M}+b+\gamma+\mu_{T}+\frac{\sigma_{1}^{2}}{2}+\frac{\sigma_{2}^{2}}{2}+\frac{\sigma_{3}^{2}}{2}+\frac{\sigma_{4}^{2}}{2}\right\} .
\end{aligned}
$$


In view of (57), we obtain that $\mathscr{L} \widehat{U} \leq-1$ for any Case 3. If $\left(B, M_{u}, M_{i}, T\right) \in \mathbb{D}_{3}$, we obtain $\left(B, M_{u}, M_{i}, T\right) \in \mathbb{D}_{2}$.

$$
\begin{aligned}
\mathscr{L} \widehat{U} \leq & -\frac{\beta M_{u} B}{M_{i}}+M \phi B+\frac{\delta B}{K}+M_{u}\left(\zeta+\eta_{3} \beta\right)+\beta B-\frac{\delta B^{\theta+2}}{2\left(\eta_{1} b+\eta_{2} \gamma\right) K}-\frac{\mu_{M}}{2}\left(M_{u}\right)^{\theta+1} \\
& -\frac{\left(b-\left(1+\left(c_{M} / 4\right)\right)\right)}{2}\left(M_{i}\right)^{\theta+1}-\frac{\mu_{T}}{8}(T)^{\theta+1}+F-\delta+\mu_{M}+b+\gamma+\mu_{T}+\frac{\sigma_{1}^{2}}{2}+\frac{\sigma_{2}^{2}}{2}+\frac{\sigma_{3}^{2}}{2}+\frac{\sigma_{4}^{2}}{2}, \\
\leq & -\frac{\beta M_{u} B}{M_{i}}+G \leq-\frac{\beta}{\epsilon}+G .
\end{aligned}
$$

By virtue of (58), we can conclude that $\mathscr{L} \widehat{U} \leq-1$ for any $\quad$ Case 4 . If $\left(B, M_{u}, M_{i}, T\right) \in \mathbb{D}_{4}$, we get $\left(B, M_{u}, M_{i}, T\right) \in \mathbb{D}_{3}$.

$$
\begin{aligned}
\mathscr{L} \widehat{U} \leq & -\frac{s_{T}}{T}+M \phi B+\frac{\delta B}{K}+M_{u}\left(\zeta+\eta_{3} \beta\right)+\beta B-\frac{\delta B^{\theta+2}}{2\left(\eta_{1} b+\eta_{2} \gamma\right) K}-\frac{\mu_{M}}{2}\left(M_{u}\right)^{\theta+1} \\
& -\frac{\left(b-\left(1+\left(c_{M} / 4\right)\right)\right)}{2}\left(M_{i}\right)^{\theta+1}-\frac{\mu_{T}}{8}(T)^{\theta+1}+F-\delta+\mu_{M}+b+\gamma+\mu_{T}+\frac{\sigma_{1}^{2}}{2}+\frac{\sigma_{2}^{2}}{2}+\frac{\sigma_{3}^{2}}{2}+\frac{\sigma_{4}^{2}}{2}, \\
\leq & -\frac{s_{T}}{T}+G \leq-\frac{s_{T}}{\epsilon}+G .
\end{aligned}
$$

In view of (59), we can deduce that $\mathscr{L} \widehat{U} \leq-1$ for any $\left(B, M_{u}, M_{i}, T\right) \in \mathbb{D}_{4}$.

Case 5. If $\left(B, M_{u}, M_{i}, T\right) \in \mathbb{D}_{5}$, then one can see that

$$
\begin{aligned}
\mathscr{L} \widehat{U} \leq & -\frac{\delta B^{\theta+2}}{4\left(\eta_{1} b+\eta_{2} \gamma\right) K}+M \phi B+\frac{\delta B}{K}+M_{u}\left(\zeta+\eta_{3} \beta\right)+\beta B-\frac{\delta B^{\theta+2}}{4\left(\eta_{1} b+\eta_{2} \gamma\right) K}-\frac{\mu_{M}}{2}\left(M_{u}\right)^{\theta+1} \\
& -\frac{\left(b-\left(1+\left(c_{M} / 4\right)\right)\right)}{2}\left(M_{i}\right)^{\theta+1}-\frac{\mu_{T}}{8}(T)^{\theta+1}+F-\delta+\mu_{M}+b+\gamma+\mu_{T}+\frac{\sigma_{1}^{2}}{2}+\frac{\sigma_{2}^{2}}{2}+\frac{\sigma_{3}^{2}}{2}+\frac{\sigma_{4}^{2}}{2}, \\
\leq & -\frac{\delta B^{\theta+2}}{4\left(\eta_{1} b+\eta_{2} \gamma\right) K}+H \leq-\frac{\delta}{4\left(\eta_{1} b+\eta_{2} \gamma\right) K} \frac{1}{\epsilon^{\theta+2}}+H,
\end{aligned}
$$

where

$$
\begin{aligned}
H= & \sup _{\left(B, M_{u}, M_{i}, T\right) \in \mathbb{R}_{+}^{4}}\left\{M \phi B+\frac{\delta B}{K}+M_{u}\left(\zeta+\eta_{3} \beta\right)+\beta B-\frac{\delta B^{\theta+2}}{4\left(\eta_{1} b+\eta_{2} \gamma\right) K}-\frac{\mu_{M}}{2}\left(M_{u}\right)^{\theta+1}\right. \\
& \left.-\frac{\left(b-\left(1+\left(c_{M} / 4\right)\right)\right)}{2}\left(M_{i}\right)^{\theta+1}-\frac{\mu_{T}}{8}(T)^{\theta+1}+F-\delta+\mu_{M}+b+\gamma+\mu_{T}+\frac{\sigma_{1}^{2}}{2}+\frac{\sigma_{2}^{2}}{2}+\frac{\sigma_{3}^{2}}{2}+\frac{\sigma_{4}^{2}}{2}\right\} .
\end{aligned}
$$


By condition (60), we get that $\mathscr{L} \widehat{U} \leq-1$ for any Case 6. If $\left(B, M_{u}, M_{i}, T\right) \in \mathbb{D}_{6}$, we get $\left(B, M_{u}, M_{i}, T\right) \in \mathbb{D}_{5}$.

$$
\begin{aligned}
\mathscr{L} \widehat{U} \leq & -\frac{\mu_{M}}{4}\left(M_{u}\right)^{\theta+1}+M \phi B+\frac{\delta B}{K}+M_{u}\left(\zeta+\eta_{3} \beta\right)+\beta B-\frac{\delta B^{\theta+2}}{2\left(\eta_{1} b+\eta_{2} \gamma\right) K}-\frac{\mu_{M}}{4}\left(M_{u}\right)^{\theta+1} \\
& -\frac{\left(b-\left(1+\left(c_{M} / 4\right)\right)\right)}{2}\left(M_{i}\right)^{\theta+1}-\frac{\mu_{T}}{8}(T)^{\theta+1}+F-\delta+\mu_{M}+b+\gamma+\mu_{T}+\frac{\sigma_{1}^{2}}{2}+\frac{\sigma_{2}^{2}}{2}+\frac{\sigma_{3}^{2}}{2}+\frac{\sigma_{4}^{2}}{2}, \\
\mathscr{L} \widehat{U} \leq & -\frac{\mu_{M}}{4}\left(M_{u}\right)^{\theta+1}+J \leq-\frac{\mu_{M}}{4} \frac{1}{\epsilon^{\theta+1}}+J,
\end{aligned}
$$

where

$$
\begin{aligned}
J= & \sup _{\left(B, M_{u}, M_{i}, T\right) \in \mathbb{R}_{+}^{4}}\left\{M \phi B+\frac{\delta B}{K}+M_{u}\left(\zeta+\eta_{3} \beta\right)+\beta B-\frac{\delta B^{\theta+2}}{2\left(\eta_{1} b+\eta_{2} \gamma\right) K}-\frac{\mu_{M}}{4}\left(M_{u}\right)^{\theta+1}\right. \\
& \left.-\frac{\left(b-\left(1+\left(c_{M} / 4\right)\right)\right)}{2}\left(M_{i}\right)^{\theta+1}-\frac{\mu_{T}}{8}(T)^{\theta+1}+F-\delta+\mu_{M}+b+\gamma+\mu_{T}+\frac{\sigma_{1}^{2}}{2}+\frac{\sigma_{2}^{2}}{2}+\frac{\sigma_{3}^{2}}{2}+\frac{\sigma_{4}^{2}}{2} \cdot\right\} .
\end{aligned}
$$

According to condition (62), we attain that $\mathscr{L} \widehat{U} \leq-1$ for $\quad$ Case 7. If $\left(B, M_{u}, M_{i}, T\right) \in \mathbb{D}_{7}$, we have any $\left(B, M_{u}, M_{i}, T\right) \in \mathbb{D}_{6}$.

$$
\begin{aligned}
\mathscr{L} \widehat{U} \leq & -\frac{\left(b-\left(1+\left(c_{M} / 4\right)\right)\right)}{4}\left(M_{i}\right)^{\theta+1}+M \phi B+\frac{\delta B}{K}+M_{u}\left(\zeta+\eta_{3} \beta\right)+\beta B-\frac{\delta B^{\theta+2}}{2\left(\eta_{1} b+\eta_{2} \gamma\right) K} \\
& -\frac{\mu_{M}}{2}\left(M_{u}\right)^{\theta+1}-\frac{\left(b-\left(1+\left(c_{M} / 4\right)\right)\right)}{4}\left(M_{i}\right)^{\theta+1}-\frac{\mu_{T}}{8}(T)^{\theta+1}+F-\delta+\mu_{M}+b+\gamma+\mu_{T}+\frac{\sigma_{1}^{2}}{2}+\frac{\sigma_{2}^{2}}{2}+\frac{\sigma_{3}^{2}}{2}+\frac{\sigma_{4}^{2}}{2}, \\
\leq & -\frac{\left(b-\left(1+\left(c_{M} / 4\right)\right)\right)}{4}\left(M_{i}\right)^{\theta+1}+N \leq-\frac{\left(b-\left(1+\left(c_{M} / 4\right)\right)\right)}{4} \frac{1}{\epsilon^{3 \theta+3}}+N .
\end{aligned}
$$

where

$$
\begin{aligned}
N= & \sup _{\left(B, M_{u}, M_{i}, T\right) \in \mathbb{R}_{+}^{4}}\left\{M \phi B+\frac{\delta B}{K}+M_{u}\left(\zeta+\eta_{3} \beta\right)+\beta B-\frac{\delta B^{\theta+2}}{2\left(\eta_{1} b+\eta_{2} \gamma\right) K}-\frac{\mu_{M}}{2}\left(M_{u}\right)^{\theta+1}\right. \\
& \left.-\frac{\left(b-\left(1+\left(c_{M} / 4\right)\right)\right)}{4}\left(M_{i}\right)^{\theta+1}-\frac{\mu_{T}}{8}(T)^{\theta+1}+F-\delta+\mu_{M}+b+\gamma+\mu_{T}+\frac{\sigma_{1}^{2}}{2}+\frac{\sigma_{2}^{2}}{2}+\frac{\sigma_{3}^{2}}{2}+\frac{\sigma_{4}^{2}}{2}\right\} .
\end{aligned}
$$

It leads to $\mathscr{L} \widehat{U} \leq-1$ for any $\left(B, M_{u}, M_{i}, T\right) \in \mathbb{D}_{7}$ if $\quad$ Case 8 . If $\left(B, M_{u}, M_{i}, T\right) \in \mathbb{D}_{8}$, we attain condition (62) is satisfied.

$$
\begin{aligned}
\mathscr{L} \widehat{U} \leq & -\frac{\mu_{T}}{16}(T)^{\theta+1}+M \phi B+\frac{\delta B}{K}+M_{u}\left(\zeta+\eta_{3} \beta\right)+\beta B-\frac{\delta B^{\theta+2}}{2\left(\eta_{1} b+\eta_{2} \gamma\right) K}-\frac{\mu_{M}}{2}\left(M_{u}\right)^{\theta+1} \\
& -\frac{\left(b-\left(1+\left(c_{M} / 4\right)\right)\right)}{2}\left(M_{i}\right)^{\theta+1}-\frac{\mu_{T}}{16}(T)^{\theta+1}+F-\delta+\mu_{M}+b+\gamma+\mu_{T}+\frac{\sigma_{1}^{2}}{2}+\frac{\sigma_{2}^{2}}{2}+\frac{\sigma_{3}^{2}}{2}+\frac{\sigma_{4}^{2}}{2}, \\
\mathscr{L} \widehat{U} \leq & -\frac{\mu_{T}}{16}(T)^{\theta+1}+O \leq-\frac{\mu_{T}}{16} \frac{1}{\epsilon^{\theta+1}}+O
\end{aligned}
$$


TABLE 2: List of parameters.

\begin{tabular}{|c|c|c|c|}
\hline Parameters & Interpretation & Values & Reference \\
\hline$\delta$ & Bacterium growth rate & $5 \times 10^{-4}$ & {$[17]$} \\
\hline$K$ & Carrying capacity & $1 \times 10^{8}$ & {$[17]$} \\
\hline$\eta_{1}$ & Death rate of an infected macrophages & 50 & {$[17]$} \\
\hline$\eta_{2}$ & Extracellular bacteria $(B)$ rate by burst & 20 & {$[17]$} \\
\hline$\eta_{3}$ & Engulfment rate of uninfected macrophages & 25 & {$[17]$} \\
\hline$c$ & Half saturation rate in uninfected macrophages & 150 & {$[17]$} \\
\hline$\zeta$ & Killing rate of extracellular bacteria & $1.25 \times 10^{-8}$ & {$[17]$} \\
\hline$s_{M}$ & Influx rate of uninfected macrophages & 5000 & [17] \\
\hline$\mu_{M}$ & Death rates of uninfected macrophages & 25 & Assumed \\
\hline$\beta$ & Infection rates of uninfected macrophages & $2 \times 10^{-7}$ & [17] \\
\hline$b$ & Killing rate of infected macrophages & $1.1 \times 10^{-4}$ & Assumed \\
\hline$\gamma$ & Cell-mediated adaptive immune response rate & $5 \times 10^{-8}$ & [17] \\
\hline$s_{T}$ & Natural recruitment rate of activated CD4+ T cells & 6.6 & {$[17]$} \\
\hline$c_{B}$ & Maximum killing rates of extracellular bacteria macrophages & $5 \times 10^{-3}$ & {$[17]$} \\
\hline$c_{M}$ & Maximum killing rates of infected macrophages & $1 \times 10^{-3}$ & {$[17]$} \\
\hline$e_{B}^{N 1}$ & Saturating factor of extracellular bacteria macrophages & $1 \times 10^{-4}$ & {$[17]$} \\
\hline$e_{M}$ & Saturating factor of infected macrophages & $1 \times 10^{-4}$ & {$[17]$} \\
\hline$\mu_{T}$ & Death rate of activated CD4+ T cells & 0.33 & [17] \\
\hline
\end{tabular}

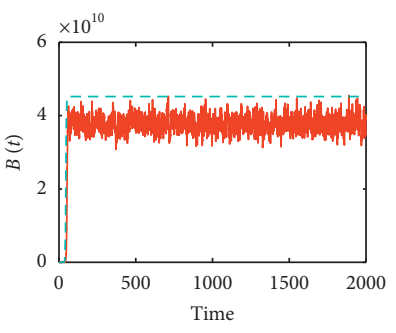

- Stochastic

- - - Deterministic

(a)

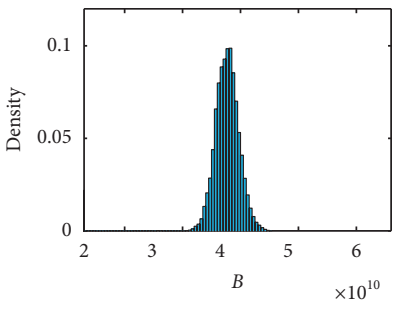

(e)

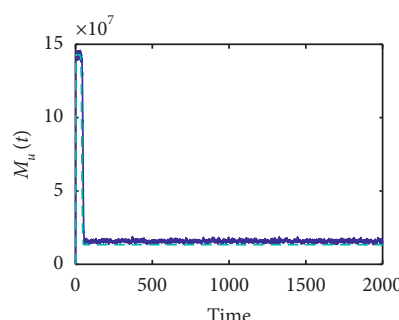

- Stochastic

(b)

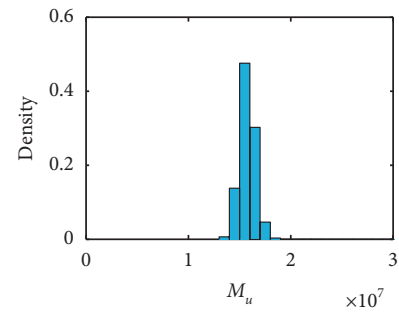

(f)

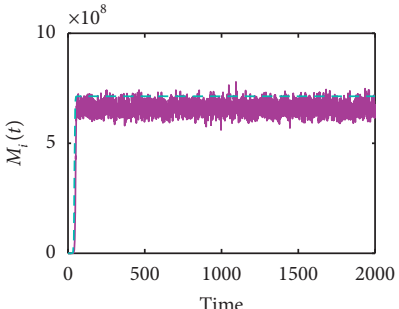

- Stochastic

(c)

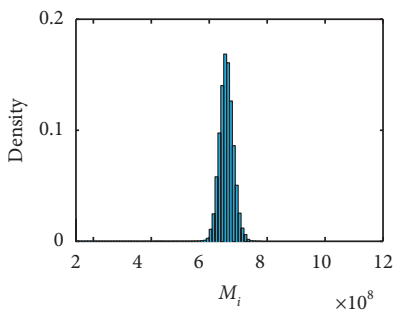

(g)

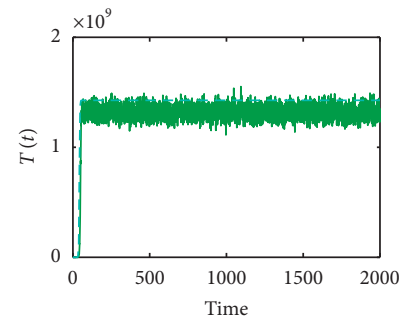

- Stochastic

- - - Deterministic

(d)

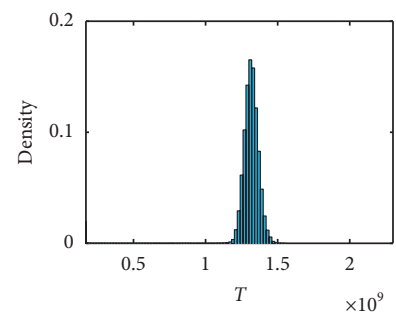

(h)

Figure 1: The first segment of the figure represents the stochastic TB system (5) which fluctuates around the endemic equilibrium of deterministic TB system (1) if the environmental white noises are sufficiently small. The second segment of the figure reflects the probability distribution of the unique ergodic stationary distribution of the stochastic TB system (5).

where

$$
\begin{aligned}
O= & \sup _{\left(B, M_{u}, M_{i}, T\right) \in \mathbb{R}_{+}^{4}}\left\{M \phi B+\frac{\delta B}{K}+M_{u}\left(\zeta+\eta_{3} \beta\right)+\beta B-\frac{\delta B^{\theta+2}}{2\left(\eta_{1} b+\eta_{2} \gamma\right) K}-\frac{\mu_{M}}{2}\left(M_{u}\right)^{\theta+1}\right. \\
& \left.-\frac{\left(b-\left(1+\left(c_{M} / 4\right)\right)\right)}{2}\left(M_{i}\right)^{\theta+1}-\frac{\mu_{T}}{16}(T)^{\theta+1}+F-\delta+\mu_{M}+b+\gamma+\mu_{T}+\frac{\sigma_{1}^{2}}{2}+\frac{\sigma_{2}^{2}}{2}+\frac{\sigma_{3}^{2}}{2}+\frac{\sigma_{4}^{2}}{2}\right\} .
\end{aligned}
$$




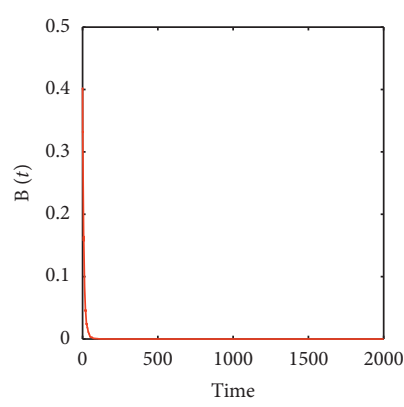

(a)

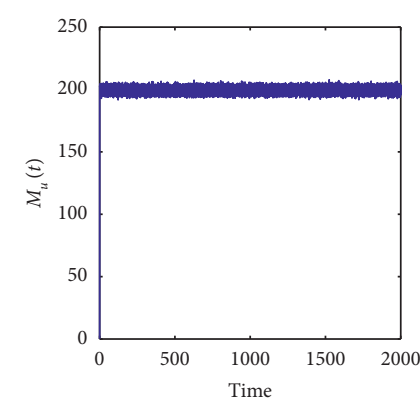

(b)

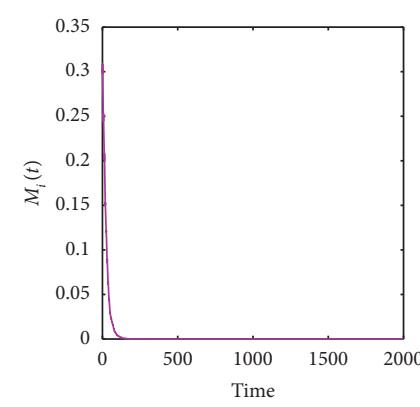

(c)

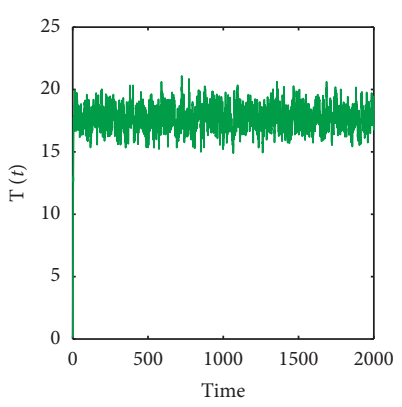

(d)

Figure 2: Simulations of the stochastic TB model (5) with initial conditions $B(0), M_{u}(0), M_{i}(0), T(0)=(0.4,0.2,0.3,0.1)$. Parameter values used are as given in Table 1.

It follows from (63) that $\mathscr{L} \widehat{U} \leq-1$ for any $\left(B, M_{u}, M_{i}\right.$, $T) \in \mathbb{D}_{8}$.

Obviously, from (64), (65), (67)-(69), (71), (73), and (75), we attain that for a sufficiently small $\epsilon, \mathscr{L} V\left(B, M_{u}\right.$, $\left.M_{i}, T\right) \leq-1, \forall\left(B, M_{u}, M_{i}, T\right) \in \mathbb{R}_{+}^{4} \backslash \mathbb{D}_{\epsilon}$. Hence, condition $A_{2}$ of Lemma 2 holds. It follows from Lemma 2 that the stochastic system (5) is ergodic and has a unique stationary distribution $\pi($.$) . This completes the proof.$

Remark 5. Notice that the expression of $\mathscr{R}_{0}^{s}$ converges with the basic reproduction number $\mathscr{R}_{0}$ for the deterministic model (1) if the environmental white noise is not taken into account. For example, we choose the real-life parameter values in Table 2, except $\delta=7.2 \times 10^{-4}, b=1.25, s_{M}=1 \times 10^{9}, \mu_{M}=7, \beta=1.5 \times$ $10^{-9}, \gamma=2 \times 10^{-2}, \quad s_{T}=2 \times 10^{-4}, c_{M}=1 \times 10^{-4}, \quad e_{M}=$ $1 \times 10^{-6}, c_{B}=5 \times 10^{-6}, e_{B}=1 \times 10^{-2}, \mu_{T}=50$ with $\sigma_{1}=$ $0=\sigma_{2}=\sigma_{3}=\sigma_{4}$. We have $\mathscr{R}_{0}=1.3507$ and $\mathscr{R}_{0}^{s}=1.3500$.

On the other hand, we select the above parameters except the environmental white noises $\sigma_{1}=0.3, \sigma_{2}=$ $0.2, \sigma_{3}=0.4$, and $\sigma_{4}=0.3$. We have $\mathscr{R}_{0}^{s}=1.2589$, and the conditions of Theorem 2 are satisfied. Figure 1 represents the stochastic TB system (5) fluctuates around the endemic equilibrium $E_{1}=\left(B_{1}, M_{u_{1}}, M_{i_{1}}, T_{1}\right)$ of deterministic TB system (1), where $B_{1}=4.5202 \times 10^{10}, M_{u_{1}}=$ $1.3368 \times 10^{7}, M_{i_{1}}=7.1372 \times 10^{8}$, and $T_{1}=1.4269 \times 10^{9}$. $\widetilde{\mathscr{R}}_{0}=\frac{4\left[\beta s_{M}+\mu_{M}\left(\delta+b N_{1}+\gamma N_{2}\right)\right]}{\mu_{M}\left(\sigma_{1}^{2} \wedge \sigma_{3}^{2}\right)} \approx \frac{0.5931}{2.2500} \approx 0.2636<1$,

\section{Numerical Simulations}

In this section, we probe the extinction of the MTB, ergodic stationary distribution of the stochastic system (5), and the effect of varied environmental noise on model dynamic behavior based on the real-life parameters of theoretical outcomes by numerical simulations. Here, we utilize Milstein's higher-order method [44] and the host-pathogen stochastic TB model can be written as the subsequent discretization equations:

$$
\begin{aligned}
& B_{k+1}=B_{k}+\left[\delta B_{k}\left(1-\frac{B_{k}}{K}\right)+M_{i_{k}}\left(\eta_{1} b+\eta_{2} \gamma \frac{T_{k}}{c+T_{k}}\right)-M_{u_{k}} B_{k}\left(\zeta+\eta_{3} \beta\right)\right] \Delta t+\sigma_{1} B_{k} \sqrt{\Delta t} \vartheta_{k}+\frac{\sigma_{1}^{2}}{2} B_{k}\left(\Delta t \vartheta_{k}^{2}-\Delta t\right), \\
& M_{u_{k+1}}=M_{u_{k}}+\left[s_{M}-\mu_{M} M_{u_{k}}-\beta M_{u_{k}} B\right] \Delta t+\sigma_{2} M_{u_{k}} \sqrt{\Delta t} \psi_{k}+\frac{\sigma_{2}^{2}}{2} M_{u_{k}}\left(\Delta t \psi_{k}^{2}-\Delta t\right), \\
& M_{i_{k+1}}=M_{i_{k}}+\left[\beta M_{u_{k}} B_{k}-b M_{i_{k}}-\gamma M_{i_{k}} \frac{T_{k}}{c+T_{k}}\right] \Delta t+\sigma_{3} M_{i_{k}} \sqrt{\Delta t} \rho_{k}+\frac{\sigma_{3}^{2}}{2} M_{i_{k}}\left(\Delta t \rho_{k}^{2}-\Delta t\right), \\
& T_{k+1}=T_{k}+\left[s_{T}+c_{M} M_{i_{k}} \frac{T_{k}}{1+e_{m} T_{k}}+c_{B} B_{k} \frac{T_{k}}{1+e_{B} T_{k}}-\mu_{T} T_{k}\right] \Delta t+\sigma_{4} T_{k} \sqrt{\Delta t} \nu_{k}+\frac{\sigma_{4}^{2}}{2} T_{k}\left(\Delta t v_{k}^{2}-\Delta t\right),
\end{aligned}
$$

where $\vartheta_{k}, \psi_{k}, \rho_{k}$, and $v_{k}, k=1,2, \ldots, n$, are the $k$ th perception of four mutually independent Gaussian random variables with $N(0,1), \sigma_{i}, i=1,2,3,4$ are the intensities of environmental noise, and $\Delta t>0$ is the increase of time. Here we choose time step $\Delta t=0.02$.

Throughout the literature, the initial values $B(0)=0.4$, $M_{u}(0)=0.2, M_{i}(0)=0.3, T(0)=0.1$ and suppose that the unit of time is two hours in a day. We choose the parameter values of deterministic TB system (1) as given in Table 2 .

Note that, $\mathscr{R}_{0}=0.2816<1$ and the deterministic TB model (1) has an infection-free equilibrium $E_{0}=\left(M_{u_{0}}, T_{0}\right.$, $\left.B_{0}, M_{i_{0}}\right)=(200,20,0,0)$, which is globally asymptotically stable on $\Gamma$. We fix the parameter values in Table 2 and intensities of environmental noise $\sigma_{1}=0.4, \sigma_{2}=0.4$, 


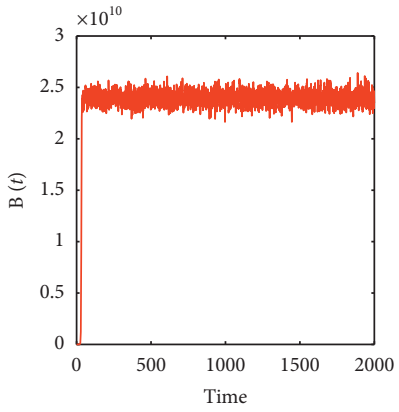

(a)

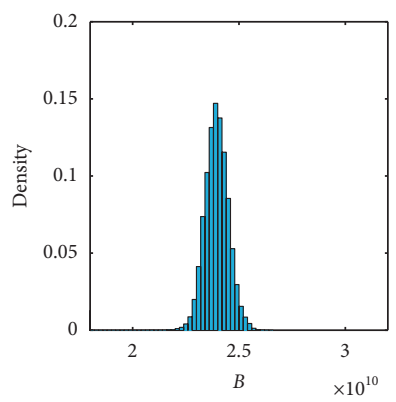

(e)

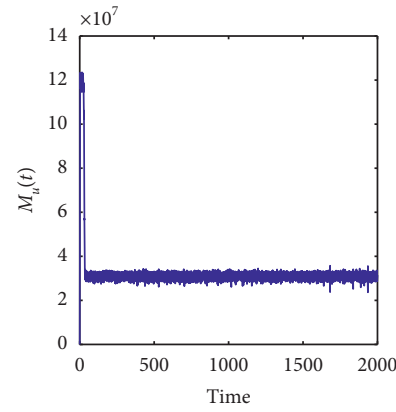

(b)

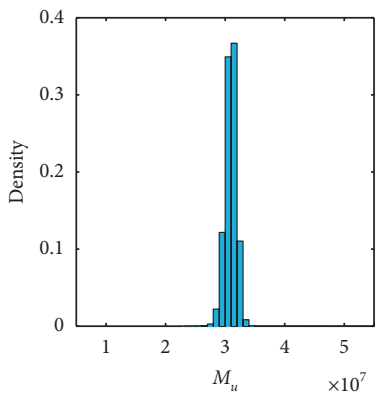

(f)

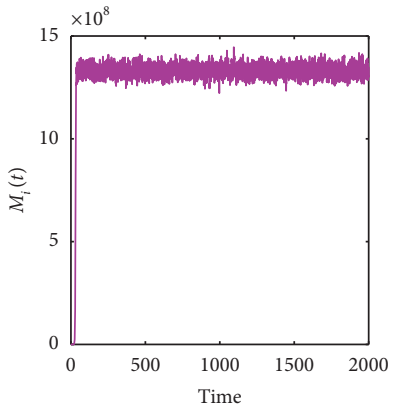

(c)

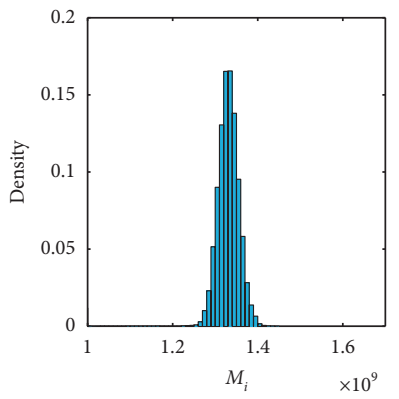

(g)

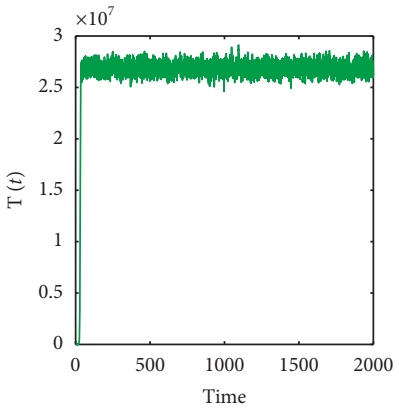

(d)

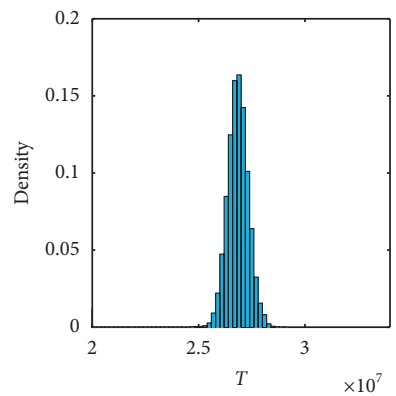

(h)

FIgURE 3: The first and second segments of the figures exhibit the existence of the unique stationary distribution of the stochastic TB model (5) and the probability distribution of the stationary distribution, respectively.

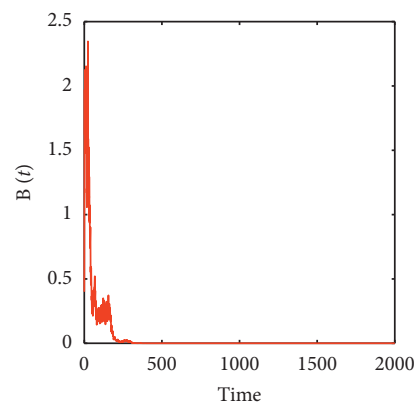

(a)

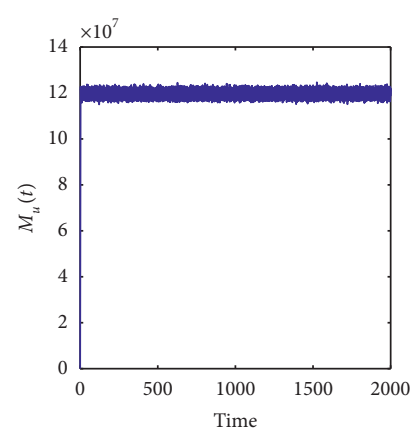

(b)

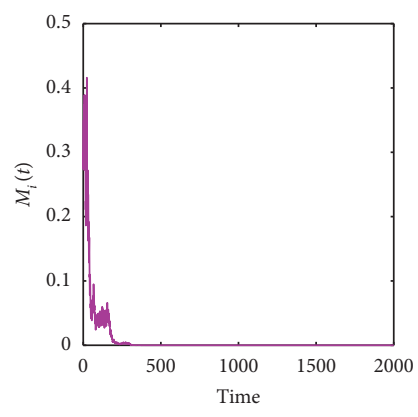

(c)

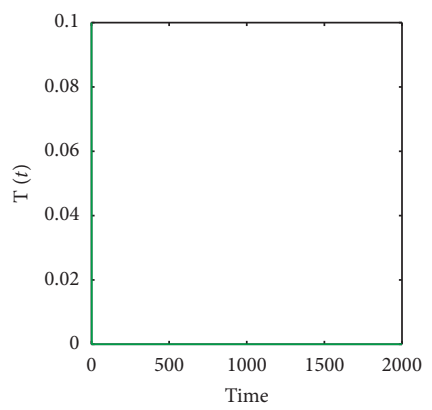

(d)

FIgURE 4: The intensities of large environmental white noise $\sigma_{1}=1.25, \sigma_{2}=0.4, \sigma_{3}=1.25$ and $\sigma_{4}=0.2$ cause extinction of the MTB.

$\sigma_{3}=0.3, \sigma_{4}=0.3$. By calculating, and $25=\mu_{M}>\left(\left(\sigma_{1}^{2} \vee\right.\right.$ $\left.\left.\sigma_{3}^{2}\right) / 2\right)=\min (0.08,0.045)=0.045$, the conditions of Theorem 2 are satisfied. Figure 2 exhibits that the population of MTB and population of infected macrophages (LTBI or active TB) of the stochastic TB system (5) to eradicate the tuberculosis bacilli germs are almost surely from the infected TB individuals at this stage.

On the other hand, increasing the infected macrophage killing rate $b$ from $1.1 \times 10^{-4}$ to 1.25 , increasing bacterium growth rate $B$, increasing the death rate of activated CD4+ $\mathrm{T}$ cells, and decreasing the natural recruitment rate of activated CD4+ T cells, etc., are vulnerable factors for tubercle bacillus which can grow from latency TB infection to oscillation and then reach as to active TB infection.

Keep all the parameter values same as in Table 2, except $\delta=5 \times 10^{-3}, s_{M}=3 \times 10^{9}, \beta=3 \times 10^{-9}, b=1.25, \gamma=0.4$,
$s_{T}=2 \times 10^{-4}, c_{B}=5 \times 10^{-6}, c_{M}=1 \times 10^{-4}, e_{B}=1 \times 10^{-2}$, $e_{M}=1 \times 10^{-4}, \mu_{T}=50$, with the intensities of environmental white noise as follows: $\sigma_{1}=0.2, \sigma_{2}=0.5, \sigma_{3}=0.2$, and $\sigma_{4}=0.1$. By computing $\mathscr{R}_{0}^{s}=\left(s_{M} \beta /\left(\vartheta+\left(\sigma_{1}^{2} / 2\right)\right)\left(\mu_{M}+\right.\right.$ $\left.\left.\left(\sigma_{2}^{2} / 2\right)\right)\left(b+\gamma+\left(\sigma_{3}^{2} / 2\right)\right)\right)\left(\eta_{1} b+\left(\eta_{2} \gamma s_{T} / s_{T}+c \mu_{T}+\left(c \sigma_{4}^{2} / 2\right)\right)\right)=$ $1.0171>1$, the conditions of Theorem 2 are fulfilled. Elseways, the deterministic TB model (1) has a unique positive infected equilibrium $E_{1}=\left(B_{1}, M_{u_{1}}, M_{i_{1}}, T_{1}\right)$, where $B_{1}=2.4718 \times 10^{10}, M_{u_{1}}=3.0256 \times 10^{7}, M_{i_{1}}=1.3598 \times 10^{9}$, and $T_{1}=2.7432 \times 10^{7}$, which is globally asymptotically stable on $\Gamma$. For the already stipulated small intensities of white noise, the stochastic TB system (5) oscillates around the positive infected equilibrium $E_{1}$. Figure 3 evinces the existence of the unique ergodic stationary distribution of the stochastic TB model (5) if $\mathscr{R}_{0}^{s}>1$, which indicates that the disease will persist and prevail in an MTB population. The 
second segment of Figure 3 evaluates the probability density distribution functions of the stationary distribution.

We keep all the parameter values as in Figure 3 with different intensities of environmental large white noise $\sigma_{1}=1.25, \sigma_{2}=0.4, \sigma_{3}=1.25$, and $\sigma_{4}=0.2$. Figure 4 reveals that the population of MTB and population of infected macrophages are swept away in the stochastic TB model (5) even while they are still persistent in the deterministic TB system (1). Hence, large intensity environmental white noise may be helpful for suppressing the disease outbreak and eradicating the MTB.

\section{Conclusions and Future Directions}

MTB is a leading communicable opportunistic disease in many countries and the main cause of chronic lung disease. In this literature, we studied the dynamical behavior of a stochastic host-pathogen tuberculosis model with adaptive immune response. Firstly, we exhibited that the stochastic TB model (5) has certain unique global positive solutions. We established sufficient conditions for the extinction of the tubercle bacillus. Also, we attained sufficient conditions for the existence of a unique ergodic stationary distribution of the positive solutions to the stochastic TB model (5) by applying a suitable Lyapunov function. Finally, the numerical simulations are provided to validate analytical outcomes and the dynamics of the stochastic host-pathogen TB model.

Certain interesting topics and practical realism earn further consideration. On the flip side, one may suggest some more realistic but complex models, such as considering the effects of impulsive perturbations on the stochastic TB system (5). The motivation for impulsive perturbations is that discontinuity is a common phenomenon and the real-life scenarios are generally discontinuous fashion like time and space. During the anti-TB treatment, pharmacokinetic variation and association of several drugs in a regimen are naturally nonlinear and occur only within some limited precise data spaces and often in a discontinuous manner [45]. Additionally, in the summer the incidence of TB is high, since it is speculated that the disease may have been obtained during the winter season. Due to air pollution, carbon monoxide stimulates bacillary reactivation and other factors such as poor water quality, soil pollution, and inadequate sanitation, which increase the risk of TB outbreaks. Owing to these sudden environmental changes, it is arousing curiosity to introduce the colored noise, for instance, continuous-time Markov chain into the stochastic TB system (5). Often, the switching among distinct environments is memoryless and the waiting time for the next switch is exponentially distributed [46, 47].

Finally, the memory CD8+ T cells can swiftly obtain effector functions to destroy infected cells and/or secrete inflammatory cytokines such as IL-2, IL-4, and IL-5 prevent replication of the host-pathogen [14]. The predominant bestow factor is that the exhibition of MHC-I molecules is nearly omnipresent, whereas MHC-II molecules are revealed on a more limited set of cells. In this connection, the memory CD8+ T cells have more opportunities to encounter antigen [14]. Hence, in the stochastic TB model (5), another compartment has to be added like immune response CD8 $+\mathrm{T}$ cells which may be more effective and efficient to eradicate the tuberculosis bacilli. We leave these explorations for our future work.

\section{Data Availability}

No data were used to support this study.

\section{Conflicts of Interest}

The authors declare that they have no conflicts of interest.

\section{Acknowledgments}

This work was jointly supported by the National Natural Science Foundation of China (61773217), the Natural Science Foundation of Hunan Province (2020JJ4054), Hunan Provincial Science and Technology Project Foundation (2019RS1033), the Scientific Research Fund of Hunan Provincial Education Department (18A013).

\section{References}

[1] World Health Organization, Global Tuberculosis Report, https:/www.who.int/tb/global-report-2019, World Health Organization, Geneva, Switzerland, 2019, https://www.who. int/tb/global-report-2019.

[2] C. D. Bourke, J. A. Berkley, and A. J. Prendergast, "Immune dysfunction as a cause and consequence of malnutrition," Trends in Immunology, vol. 37, no. 6, pp. 386-398, 2016.

[3] N. Joshi, J. M. Walter, and A. V. Misharin, "Alveolar macrophages,” Cellular Immunology, vol. 330, pp. 86-90, 2018.

[4] L. Ramakrishnan, "Revisiting the role of the granuloma in tuberculosis," Nature Reviews Immunology, vol. 12, no. 5, pp. 352-366, 2012.

[5] G. Lugo-Villarino, D. Hudrisier, A. Benard, and O. Neyrolles, "Emerging trends in the formation and function of tuberculosis granulomas," Frontiers in Immunology, vol. 3, pp. 1-9, 2013.

[6] K. A. McDonough, Y. Kress, and B. R. Bloom, "Pathogenesis of tuberculosis: interaction of Mycobacterium tuberculosis with macrophages," Infection and Immunity, vol. 61, no. 7, pp. 2763-2773, 1993.

[7] W. J. Kent, Clinical Tuberculosis: A Practical Hand Book (1737), CRC Press, Boca Raton, FL, USA, 2015.

[8] V. Katalinic-Jankovic, L. Furci, and D. Maria Cirillo, "Microbiology of mycobacterium tuberculosis and a new diagnostic test for TB," in Tuberculosis, European Respiratory Monograph 58 (01-13), C. Lange and G. B. Migliori, Eds., Page Bros Ltd, Norwich, UK, 2012.

[9] J. A. Caminero, G. Sotgiu, A. Zumla, and G. B. Migliori, "Best drug treatment for multidrug-resistant and extensively drugresistant tuberculosis," The Lancet Infectious Diseases, vol. 10, no. 9, pp. 621-629, 2010.

[10] S. Dorman and A. Gupta, "Treatment of pulmonary tuberculosis," in Handbook of Tuberculosis (35-90), J. H. Grosset and R. E. Chaisson, Eds., Springer Nature, Switzerland, Germany, 2017.

[11] B. D. L. Marshall and S. Galea, "Formalizing the role of agentbased modeling in causal inference and epidemiology," American Journal of Epidemiology, vol. 181, no. 2, pp. 92-99, 2015. 
[12] M. Henao-Tamayo, D. J. Ordway, and I. M. Orme, "Memory T cell subsets in tuberculosis: what should we be targeting?" Tuberculosis, vol. 94, no. 5, pp. 455-461, 2014.

[13] T. Prezzemolo, G. Guggino, M. P. La Manna, D. Di Liberto, F. Dieli, and N. Caccamo, "Functional signatures of human CD4 and CD8T cell responses to Mycobacterium tuberculosis," Frontiers in Immunology, vol. 5, pp. 1-13, 2014.

[14] S. M. Kaech, E. J. Wherry, and R. Ahmed, "Effector and memory T-cell differentiation: implications for vaccine development," Nature Reviews Immunology, vol. 2, no. 4, pp. 251-262, 2002.

[15] A. Kallies, "Distinct regulation of effector and memory T-cell differentiation," Immunology \& Cell Biology, vol. 86, no. 4, pp. 325-332, 2008.

[16] D. A. A. Vignali, L. W. Collison, and C. J. Workman, "How regulatory T cells work," Nature Reviews Immunology, vol. 8, no. 7, pp. 523-532, 2008.

[17] W. Zhang, "Analysis of an in-host tuberculosis model for disease control," Applied Mathematics Letters, vol. 99, Article ID 105983, 2020.

[18] M. Pitchaimani and S. P. Rajasekar, "Global analysis of stochastic SIR model with variable diffusion rates," Tamkang Journal of Mathematics, vol. 49, no. 2, pp. 155-182, 2018.

[19] S. P. Rajasekar, M. Pitchaimani, and Q. Zhu, "Dynamic threshold probe of stochastic SIR model with saturated incidence rate and saturated treatment function," Physica A: Statistical Mechanics and Its Applications, vol. 535, Article ID 122300, 2019.

[20] S. P. Rajasekar, M. Pitchaimani, and Q. Zhu, "Progressive dynamics of a stochastic epidemic model with logistic growth and saturated treatment," Physica A: Statistical Mechanics and Its Applications, vol. 538, Article ID 122649, 2020.

[21] A. Ibeas, M. de la Sen, and S. Alonso-Quesada, "Robust sliding control of SEIR epidemic models," Mathematical Problems in Engineering, vol. 2014, Article ID 104764, 14 pages, 2014.

[22] C. Ji, "The threshold for a stochastic HIV-1 infection model with Beddington-DeAngelis incidence rate," Applied Mathematical Modelling, vol. 64, pp. 168-184, 2018.

[23] X. Zhang, "Global dynamics of a stochastic avian-human influenza epidemic model with logistic growth for avian population," Nonlinear Dynamics, vol. 90, no. 4, pp. 2331-2343, 2017.

[24] Q. Liu, D. Jiang, T. Hayat, B. Ahmad, and A. Ahmed, "Stationary distribution and extinction of a stochastic SIRI epidemic model with relapse," Stochastic Analysis and Applications, vol. 36, no. 1, pp. 138-151, 2018.

[25] Z. Shi, X. Zhang, and D. Jiang, "Dynamics of an avian influenza model with half-saturated incidence," Applied Mathematics and Computation, vol. 355, pp. 399-416, 2019.

[26] Q. Zhu, "Stability analysis of stochastic delay differential equations with Lévy noise," Systems \& Control Letters, vol. 118, pp. 62-68, 2018.

[27] Q. Zhu and H. Wang, "Output feedback stabilization of stochastic feedforward systems with unknown control coefficients and unknown output function," Automatica, vol. 87, pp. 166-175, 2018.

[28] Q. Zhu, "Stabilization of stochastic nonlinear delay systems with exogenous disturbances and the event-triggered feedback control," IEEE Transactions on Automatic Control, vol. 64, no. 9, pp. 3764-3771, 2019.

[29] W. Hu, Q. Zhu, and H. R. Karimi, "Some improved razumikhin stability criteria for impulsive stochastic delay differential systems," IEEE Transactions on Automatic Control, vol. 64, no. 12, pp. 5207-5213, 2019.

[30] K. Wu and X. Ding, "Stability and stabilization of impulsive stochastic delay differential equations," Mathematical Problems in Engineering, vol. 2012, Article ID 176375, 17 pages, 2012.

[31] Q. Liu, D. Jiang, N. Shi, T. Hayat, and A. Alsaedi, "Dynamics of a stochastic tuberculosis model with constant recruitment and varying total population size," Physica A: Statistical Mechanics and Its Applications, vol. 469, pp. 518-530, 2017.

[32] Q. Liu, D. Jiang, T. Hayat, A. Alsaedi, and A. Ahmed, "Dynamics of a stochastic tuberculosis model with antibiotic resistance," Chaos, Solitons \& Fractals, vol. 109, pp. 223-230, 2018.

[33] M. Z. Xin and B. G. Wang, "Stationary distribution and extinction of a stochastic tuberculosis model," Physica A: Statistical Mechanics and Its Applications, vol. 545, Article ID 123741, 2019.

[34] F. Tao and Z. Qiu, "Global analysis of a stochastic TB model with vaccination and treatment," Discrete and Continuous Dynamical Systems Series B, vol. 24, pp. 2923-2939, 2019.

[35] Q. Liu and D. Jiang, "The dynamics of a stochastic vaccinated tuberculosis model with treatment," Physica A: Statistical Mechanics and Its Applications, vol. 527, Article ID 121274, 2019.

[36] K. Hattaf, M. Mahrouf, J. Adnani, and N. Yousfi, "Qualitative analysis of a stochastic epidemic model with specific functional response and temporary immunity," Physica A: Statistical Mechanics and Its Applications, vol. 490, pp. 591-600, 2018.

[37] K. Hattaf and N. Yousfi, "A class of delayed viral infection models with general incidence rate and adaptive immune response," International Journal of Dynamics and Control, vol. 4, no. 3, pp. 254-265, 2016.

[38] S. P. Rajasekar and M. Pitchaimani, "Qualitative analysis of stochastically perturbed SIRS epidemic model with two viruses," Chaos, Solitons \& Fractals, vol. 118, pp. 207-221, 2019.

[39] S. P. Rajasekar and M. Pitchaimani, "Ergodic stationary distribution and extinction of a stochastic SIRS epidemic model with logistic growth and nonlinear incidence," Applied Mathematics and Computation, vol. 377, Article ID 125143, 2020.

[40] X. Mao, Stochastic Differential Equations and Applications, Horwood Publishing, Chichester, UK, 2007.

[41] M. D. Iseman, "Tuberculosis therapy: past, present and future," European Respiratory Journal, vol. 20, no. 36, pp. 87S-94s, 2002.

[42] K. Dheda, G. Theron, J. G. Peter, G. Symons, R. Dawson, and P. Willcox, "TB drug resistance in high-incidence countries," Tuberculosis, vol. 58, pp. 95-110, 2012.

[43] H. minskii, Stochastic Stability of Differential Equations, Cambridge University Press, Cambridge, UK, 1980.

[44] D. J. Higham., "An algorithmic introduction to numerical simulation of stochastic differential equations," SIAM Review, vol. 43, no. 3, pp. 525-546, 2001.

[45] J. G. Pasipanodya and T. Gumbo, "Individualizing tuberculosis (TB) treatment: are TB programs in high burden settings ready for prime time therapeutic drug monitoring?" Clinical Infectious Diseases, vol. 67, no. 5, pp. 717-718, 2018.

[46] L. Qi and X. Mao, "Stochastic population dynamics under regime switching," Journal of Mathematical Analysis and Applications, vol. 334, pp. 69-84, 2007.

[47] X. Mao and C. Yuan, Stochastic Differential Equations with Markovian Switching, Imperial College Press, London, UK, 2006. 